\title{
Magnetic field induced tunability of spin Hamiltonians: Resonances and Efimov states in $\mathbf{Y b}_{2} \mathbf{T i}_{2} \mathbf{O}_{7}$
}

\author{
Yasuyuki Kato $\odot,{ }^{1}$ Shang-Shun Zhang, ${ }^{2}$ Yusuke Nishida $\odot,{ }^{3}$ and C. D. Batista $\odot^{2,4}$ \\ ${ }^{1}$ Department of Applied Physics, The University of Tokyo, Tokyo 113-8656, Japan \\ ${ }^{2}$ Department of Physics and Astronomy, University of Tennessee, Knoxville, Tennessee 37996-1200, USA \\ ${ }^{3}$ Department of Physics, Tokyo Institute of Technology, Ookayama, Meguro, Tokyo 152-8551, Japan \\ ${ }^{4}$ Neutron Scattering Division and Shull-Wollan Center, Oak Ridge National Laboratory, Oak Ridge, Tennessee 37831, USA
}

(Received 14 March 2020; revised 5 June 2020; accepted 15 June 2020; published 6 July 2020)

\begin{abstract}
Universality is a powerful concept that arises from the divergence of a characteristic length scale. For condensed matter systems, this length scale is typically the correlation length, which diverges at critical points separating two different phases. Few-particle systems exhibit a simpler form of universality when the $s$-wave scattering length diverges. A prominent example of universal phenomena is the emergence of an infinite tower of three-body bound states obeying discrete scale invariance, known as the Efimov effect, which has been subject to extensive research in chemical, atomic, nuclear, and particle physics. In principle, these universal phenomena can also emerge in the excitation spectrum of condensed matter systems, such as quantum magnets [Y. Nishida, Y. Kato, and C. Batista, Nat. Phys. 9, 93 (2013)]. However, the limited tunability of the effective interparticle interaction relative to the kinetic energy has precluded so far their observation. Here, we demonstrate that a high degree of magnetic field induced tunability can also be achieved in quantum magnets with strong spin-orbit coupling: A two-magnon resonance condition can be achieved in $\mathrm{Yb}_{2} \mathrm{Ti}_{2} \mathrm{O}_{7}$ with a field of $\sim 13 \mathrm{~T}$ along the [110] direction, which leads to the formation of Efimov states in the three-magnon spectrum of this material. Raman scattering experiments can reveal the field-induced two-magnon resonance, as well as the Efimov three-magnon bound states that emerge near the resonance condition.
\end{abstract}

DOI: 10.1103/PhysRevResearch.2.033024

\section{INTRODUCTION}

The simplest example of universality arises in the vicinity of scattering resonances of few-body systems, where the lowenergy physics is characterized solely by the $s$-wave scattering length $a$. One of the most prominent observations of universal phenomena at $a \rightarrow \infty$ is the emergence of an infinite tower of three-body bound states obeying discrete scale invariance, known as the Efimov effect [1],

$$
\frac{E_{n+1}}{E_{n}} \rightarrow \lambda^{-2} \quad(n \rightarrow \infty)
$$

with the universal scale factor $\lambda=22.6944$. For the last five decades, this effect has been subject to extensive research in chemical, atomic, nuclear, and particle physics [2-8]. However, Efimov states have been observed in very limited systems due to the requirement of proximity to a scattering resonance. Thus, the tunability of interparticle interactions is highly desired for their realization, which has only been achieved for atomic gases. As we will demonstrate here, this tunability can also be achieved in quantum magnets with strong spin-orbit coupling, which opens the possibility of

Published by the American Physical Society under the terms of the Creative Commons Attribution 4.0 International license. Further distribution of this work must maintain attribution to the author(s) and the published article's title, journal citation, and DOI. studying and observing Efimov states in condensed matter systems.

In general, the main obstacle for observing universal behavior in the vicinity of scattering resonances in condensed matter systems is their limited tunability in comparison to ultracold atoms, whose Feshbach resonances provide a way to vary $a$ by applying a uniform magnetic field [9]. Among multiple uses, this tool has served to study the crossover between Bose-Einstein condensates (BECs) of fermionic molecules and the BCS regime of weakly interacting fermion pairs in Fermi clouds [10-16]. For the BECs, Feshbach resonances have been used to study a variety of systems from the noninteracting ideal Bose gases to the unitary regime of interactions [17-26]. Is it then possible to find a counterpart of the Feshbach resonances in solid state physics?

In this paper, we provide an affirmative answer to this question by demonstrating that a uniform magnetic field can also be used to tune the $s$-wave scattering length for the collision between magnons of quantum magnets with strong spin-orbit coupling. This goal is achieved by tuning the effective magnon tunneling with the external magnetic field, while keeping the attractive magnon-magnon interaction practically unchanged. This tunability makes it possible to drive the system into its universal regime by approaching the resonance condition. Because magnons obey bosonic statistics, this is enough to realize Efimov states in the three-magnon spectrum of quantum magnets [27], such as $\mathrm{Yb}_{2} \mathrm{Ti}_{2} \mathrm{O}_{7}$, as well as other consequences of the universality. 
(a)

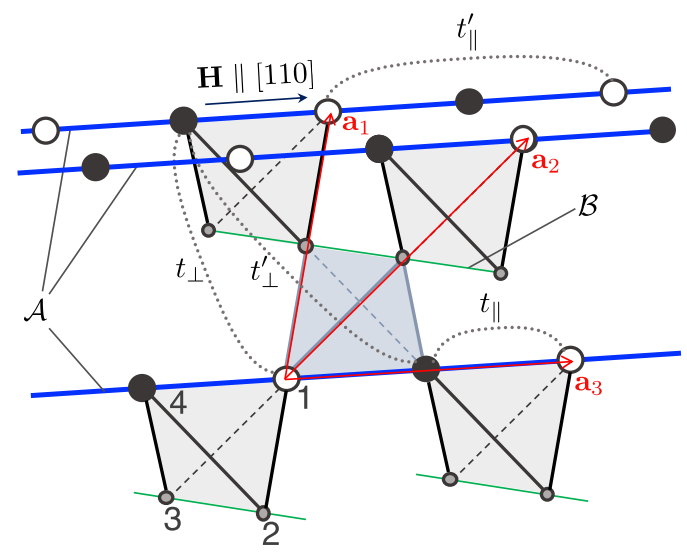

(b)

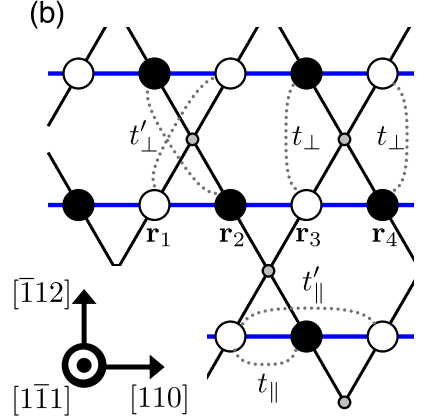

(c)

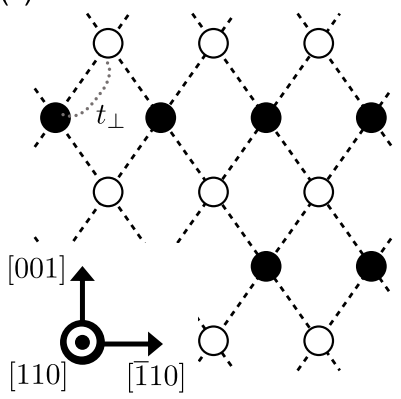

(d)

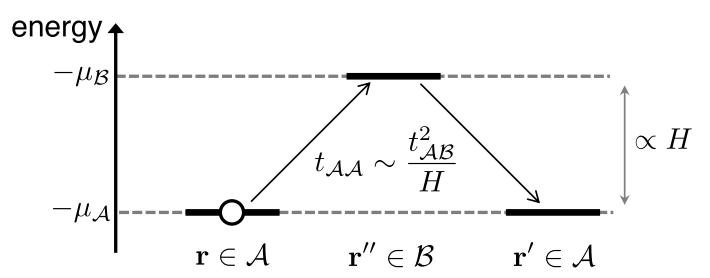

FIG. 1. Pyrochlore lattice formed by $\mathrm{Yb}$ cations. (a) Circles represent $\mathrm{Yb}$ cations. Primitive lattice vectors $\mathbf{a}_{1-3}$, sublattice index $\alpha=1-4$, and the external field direction $\mathbf{H} \|[110]$. Thick blue lines and thin green lines indicate the low-energy $\mathcal{A}$ chains of $\alpha=1$ and 4 and the high-energy $\mathcal{B}$ chains of $\alpha=2$ and 3 , respectively. (b) (11 1 ) plane of the lattice. (c) (110) plane of the lattice. Hopping paths of $t_{\|}, t_{\|}^{\prime}, t_{\perp}$, and $t_{\perp}^{\prime}$ in $\mathcal{H}_{\text {eff }}$ are indicated. The site indices $\mathbf{r}_{1-4}$ represent an example of $\left\langle\mathbf{r}_{1} \mathbf{r}_{2} \mathbf{r}_{3}\right\rangle$ or $\left\langle\mathbf{r}_{1} \mathbf{r}_{2} \mathbf{r}_{3} \mathbf{r}_{4}\right\rangle$ in $\mathcal{H}_{\text {eff }}$. (d) Schematic picture of effective hopping $t_{\mathcal{A} \mathcal{A}}$ induced by $t_{\mathcal{A B}} \cdot \mu_{\mathcal{A}}$ and $\mu_{\mathcal{B}}$ represent the chemical potential of the low-energy and high-energy sublattices, respectively.

Similar to the case of atomic gases, an external magnetic field works as an effective chemical potential for the magnons of the fully polarized magnetic ground state that is induced above the saturation field [28]. A key observation here is that the chemical potential can be made inhomogeneous in magnets with strong spin-orbit coupling and more than one magnetic ion per unit cell. For instance, $\mathrm{Yb}_{2} \mathrm{Ti}_{2} \mathrm{O}_{7}$ comprises a pyrochlore lattice (Fig. 1) of $\mathrm{Yb}^{3+}$ cations that can be divided into four symmetry-related sublattices, 1, 2, 3, and 4 corresponding to the four corners of each tetrahedron with

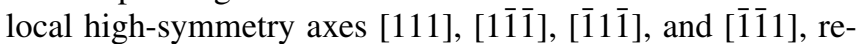
spectively. Because the effective $g$-tensor of each magnetic ion is strongly anisotropic, it has a strong sublattice dependence in a global reference frame. In other words, the chemical potential induced by an external field $\mathbf{H}=\left(H^{x}, H^{y}, H^{z}\right)$ is sublattice dependent. For $\mathbf{H} \|$ [110], the four sublattices are divided into two pairs: the low-energy $\mathcal{A}$ sublattices 1 and 4 with chemical potential $\mu_{\mathcal{A}}$ and the high-energy $\mathcal{B}$ sublattices 2 and 3 with chemical potential $\mu_{\mathcal{B}}$. As it is indicated in Fig. 1(a), the magnetic ions in the $\mathcal{A}(\mathcal{B})$ sublattices form

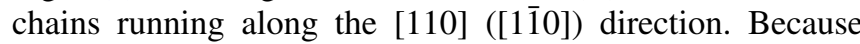
$\mu_{\mathcal{A}} \gg \mu_{\mathcal{B}}$ for large enough magnetic field values, the $\mathcal{A}$ $(\mathcal{B})$ chains become low-energy (high-energy) chains for $H \equiv$ $|\mathbf{H}| \rightarrow \infty$. Given that the $\mathcal{A}$ and $\mathcal{B}$ sublattices form a bipartite graph (bare magnon tunneling $t_{\mathcal{A B}}$ only exists between $\mathcal{A}$ and $\mathcal{B}$ sublattices), the effective magnon tunneling $t_{\mathcal{A} \mathcal{A}}$ between different low-energy chains can be continuously suppressed by increasing the energy difference $\left|\mu_{\mathcal{A}}-\mu_{\mathcal{B}}\right|$ [Fig. 1(d)]. Since $\mu_{\mathcal{A}}-\mu_{\mathcal{B}}$ is roughly proportional to $H$ and $t_{\mathcal{A} \mathcal{A}} \sim$ $t_{\mathcal{A B}}^{2} / H$, the field can be used to vary the effective magnon tunneling between different low-energy chains. In particular, the original three-dimensional system becomes quasi-onedimensional in the large field limit. While the above-described setup is the one that will be used in this paper, we note that it is also possible to make the system quasi-two-dimensional by applying the field along the [111] direction. In this case, the $\mathcal{A}$ and $\mathcal{B}$ subsystems correspond to alternating triangular and kagome layers, respectively.

The structure of this paper is as follows. In Sec. II, we introduce the low-energy effective hard-core boson model derived from the effective spin model of $\mathrm{Yb}_{2} \mathrm{Ti}_{2} \mathrm{O}_{7}$. In addition, we describe the numerical calculation methods to solve the two- and three-magnon problems. In Sec. III, we show the results of analytical and numerical calculations of the one-, two-, and three-magnon problems. We demonstrate that the magnon scattering length can be tuned with an external magnetic field producing a two-magnon resonance condition for a field strength of $\sim 13 \mathrm{~T}$ along the [110] direction. We also demonstrate that Efimov states emerge in the three-magnon sector near the two-magnon resonance condition. Finally, in Sec. IV, we summarize the main results and discuss their experimental realization. All technical details are presented in the Appendixes.

\section{MODEL AND METHOD}

\section{A. Effective hard-core boson model}

$\mathrm{Yb}_{2} \mathrm{Ti}_{2} \mathrm{O}_{7}$ comprises a pyrochlore lattice of magnetic $\mathrm{Yb}^{3+}$ ions [Fig. 1(a)], whose low-energy degrees of freedom (doublets) are described by an effective spin- $1 / 2$ Hamiltonian [29,30],

$$
\begin{aligned}
\mathcal{H}_{\text {spin }}= & \sum_{\left\langle\mathbf{r} \mathbf{r}^{\prime}\right\rangle}\left[J_{z z} \mathrm{~S}_{\mathbf{r}}^{z} \mathbf{S}_{\mathbf{r}^{\prime}}^{z}-J_{ \pm}\left(\mathrm{S}_{\mathbf{r}}^{+} \mathbf{S}_{\mathbf{r}^{\prime}}^{-}+\text {H.c. }\right)\right. \\
& +J_{ \pm \pm}\left(\gamma_{\alpha_{\mathbf{r}} \alpha_{\mathbf{r}^{\prime}}} S_{\mathbf{r}}^{+} \mathbf{S}_{\mathbf{r}^{\prime}}^{+}+\text {H.c. }\right) \\
& \left.+J_{z \pm}\left\{\mathbf{S}_{\mathbf{r}}^{z}\left(\zeta_{\alpha_{\mathbf{r}} \alpha_{\mathbf{r}^{\prime}}} \mathrm{S}_{\mathbf{r}^{\prime}}^{+}+\text {H.c. }\right)+\left(\mathbf{r} \leftrightarrow \mathbf{r}^{\prime}\right)\right\}\right] \\
& -\mu_{\mathrm{B}} \mu_{0} \sum_{\eta, v} H^{\eta} \sum_{\mathbf{r}} g_{\alpha_{\mathbf{r}}}^{\eta \nu} S_{\mathbf{r}}^{\nu} .
\end{aligned}
$$

The spin- $1 / 2$ operators $\mathbf{S}_{\mathbf{r}}^{v}\left(v=x, y\right.$, or $z$, and $\mathbf{S}_{\mathbf{r}}^{ \pm}=\mathrm{S}_{\mathbf{r}}^{x} \pm$ $i \mathrm{~S}_{\mathbf{r}}^{y}$ ) are expressed in a sublattice-dependent reference frame, whose local $z$ axis is parallel to the local [111] direction. The index $\alpha_{\mathbf{r}}=1-4$ indicates the sublattice of the site $\mathbf{r}$, and $\left\langle\mathbf{r r}^{\prime}\right\rangle$ indicates that the sum $\sum_{\left\langle\mathbf{r r}^{\prime}\right\rangle}$ runs over the nearest- 
neighbor sites of the pyrochlore lattice. The indices of $\eta$ and $v$ run over $x, y$, and $z . \mu_{\mathrm{B}}$ is the Bohr magneton, and $\mu_{0}$ is the permeability constant. $\gamma_{\alpha_{\mathrm{r}} \alpha_{\mathrm{r}^{\prime}}}$ and $\zeta_{\alpha_{\mathrm{r}} \alpha_{\mathrm{r}^{\prime}}}$ are phase factors, and $g_{\alpha_{\mathrm{r}}}$ is the $g$-tensor for sites in the sublattice $\alpha_{\mathbf{r}}$ (Appendix A). The model parameters $\left(J_{z z}, J_{ \pm \pm}, J_{ \pm}, J_{z \pm}\right.$, $g_{\|}$, and $\left.g_{\perp}\right)$ are set to the values estimated from a recent inelastic neutron scattering measurement of $\mathrm{Yb}_{2} \mathrm{Ti}_{2} \mathrm{O}_{7}$ [31]. The effective spin- $1 / 2$ moments can be mapped into hard-core bosons with creation and annihilation operators $a_{\mathrm{r}}^{\dagger}$ and $a_{\mathrm{r}}$, respectively [32]. By choosing the local quantization axis to be parallel to the magnetic moment, the exact mapping leads to a hard-core boson model with no linear terms in the creation or annihilation operators (Appendix B).

The Zeeman term, which is dominant for relatively high fields $\left(\mu_{0} H \gg\left|J_{z z}\right|,\left|J_{ \pm}\right|,\left|J_{ \pm \pm}\right|,\left|J_{z \pm}\right|\right)$, becomes a sublatticedependent chemical potential term in the hard-core boson language, with $\left|\mu_{\alpha}\right|$ being roughly proportional to $H$. In particular, the difference between $\mu_{1}=\mu_{4}$ and $\mu_{2}=\mu_{3}$ increases in proportion to $H$ because of the strongly anisotropic $g$-tensor that arises from a combination of strong spin-orbit coupling and the crystal field of the $\mathrm{Yb}$ cation. The energy scale of all the other terms is much smaller than $\left|\mu_{\alpha}\right|$ and $\left|\mu_{1}-\mu_{2}\right|$ for high enough fields. Thus, we will regard the chemical potential (Zeeman) terms as the unperturbed Hamiltonian and we will treat the rest as perturbation.

By applying the second-order degenerate perturbation theory (Appendix C), we obtain an effective low-energy Hamiltonian for bosons on the chains 1 and 4 that conserves the particle number,

$$
\begin{aligned}
& \mathcal{H}_{\text {eff }}=-\mu \sum_{\mathbf{r}} n_{\mathbf{r}}+t_{\|} \sum_{\left\langle\mathbf{r r}^{\prime}\right\rangle_{\|}}\left(a_{\mathbf{r}^{\dagger}}^{\dagger} a_{\mathbf{r}^{\prime}}+\text { H.c. }\right) \\
& +t_{\|}^{\prime} \sum_{\left\langle\mathbf{r r}^{\prime}\right\rangle_{\|}}\left(a_{\mathbf{r}}^{\dagger} a_{\mathbf{r}^{\prime}}+\text { H.c. }\right)+t_{\perp} \sum_{\left\langle\mathbf{r r}^{\prime}\right\rangle_{\perp}}\left(a_{\mathbf{r}}^{\dagger} a_{\mathbf{r}^{\prime}}+\text { H.c. }\right) \\
& +t_{\perp}^{\prime} \sum_{\left\langle\mathbf{r r}^{\prime}\right\rangle_{\perp}^{\prime}}\left(a_{\mathbf{r}}^{\dagger} a_{\mathbf{r}^{\prime}}+\text { H.c. }\right)+u_{\|} \sum_{\left\langle\mathbf{r} \mathbf{r}^{\prime}\right\rangle_{\|}} n_{\mathbf{r}} n_{\mathbf{r}^{\prime}} \\
& +u_{\|}^{\prime} \sum_{\left\langle\mathbf{r} \mathbf{r}^{\prime}\right\rangle_{\|}^{\prime}} n_{\mathbf{r}} n_{\mathbf{r}^{\prime}}+u_{\perp} \sum_{\left\langle\mathbf{r} \mathbf{r}^{\prime}\right\rangle_{\perp}} n_{\mathbf{r}} n_{\mathbf{r}^{\prime}} \\
& +u_{\perp}^{\prime} \sum_{\left\langle\mathbf{r} \mathbf{r}^{\prime}\right\rangle_{\perp}^{\prime}} n_{\mathbf{r}} n_{\mathbf{r}^{\prime}}+v_{1} \sum_{\left\langle\mathbf{r}_{1} \mathbf{r}_{2} \mathbf{r}_{3}\right\rangle}\left(a_{\mathbf{r}_{1}}^{\dagger} n_{\mathbf{r}_{2}} a_{\mathbf{r}_{3}}+\text { H.c. }\right) \\
& +v_{2} \sum_{\left\langle\mathbf{r}_{1} \mathbf{r}_{2} \mathbf{r}_{3} \mathbf{r}_{4}\right\rangle}\left(a_{\mathbf{r}_{2}}^{\dagger} a_{\mathbf{r}_{3}}+\text { H.c. }\right)\left(n_{\mathbf{r}_{1}}+n_{\mathbf{r}_{4}}\right) \\
& +w \sum_{\left\langle\mathbf{r}_{1} \mathbf{r}_{2} \mathbf{r}_{3}\right\rangle} n_{\mathbf{r}_{1}} n_{\mathbf{r}_{2}} n_{\mathbf{r}_{3}}+U \sum_{\mathbf{r}} n_{\mathbf{r}}\left(n_{\mathbf{r}}-1\right),
\end{aligned}
$$

where $t_{\perp}^{\prime}=t_{\perp}$ and $u_{\perp}^{\prime}=-u_{\perp} . \mu$ is the chemical potential including the second-order correction, the following four hopping terms represent the kinetic energy, and the rest of the terms are multibody interactions. The brackets $\left\langle\mathbf{r} \mathbf{r}^{\prime}\right\rangle_{\|},\left\langle\mathbf{r r}^{\prime}\right\rangle_{\|}^{\prime}$, $\left\langle\mathbf{r} \mathbf{r}^{\prime}\right\rangle_{\perp}$, and $\left\langle\mathbf{r r}^{\prime}\right\rangle_{\perp}^{\prime}$ indicate that the sums run over intrachain nearest neighbors (NN), intrachain next-NN, interchain $\mathrm{NN}$, and interchain next-NN separated by a site on sublattices 2 or 3, respectively [Figs. 1(a) and 1(b)]. The brackets $\left\langle\mathbf{r}_{1} \mathbf{r}_{2} \mathbf{r}_{3}\right\rangle$ and $\left\langle\mathbf{r}_{1} \mathbf{r}_{2} \mathbf{r}_{3} \mathbf{r}_{4}\right\rangle$ indicate that the corresponding sums run over all possible combinations of three and four consecutive sites,
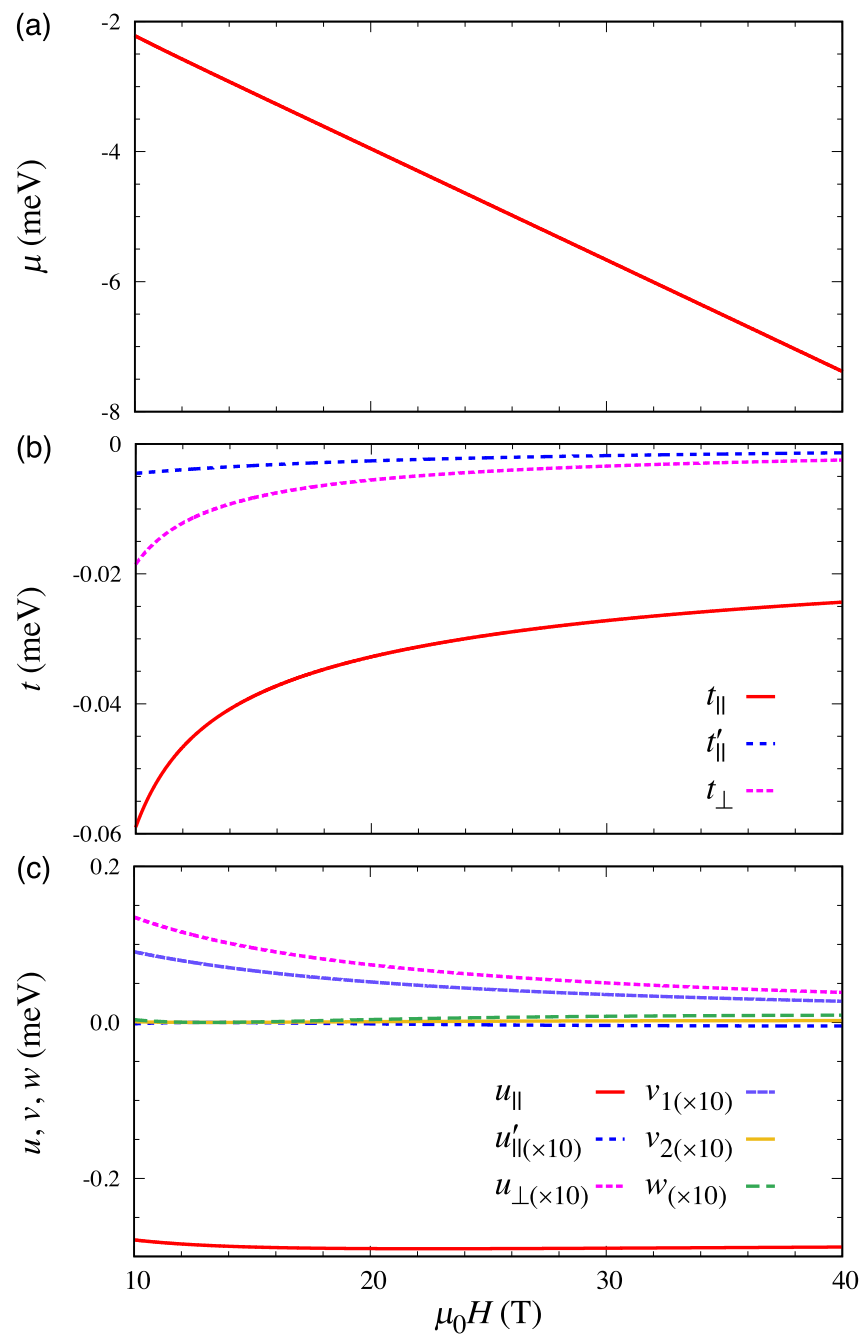

FIG. 2. Model parameters for the effective boson Hamiltonian $\mathcal{H}_{\text {eff }}$ obtained for the spin Hamiltonian $\mathcal{H}_{\text {spin }}$ reported in Ref. [31]. (a) Chemical potential $\mu$. (b) Hopping amplitudes $t_{\|}, t_{\|}^{\prime}$, and $t_{\perp}$. (c) Interactions $u_{\|}, u_{\|}^{\prime}, u_{\perp}, v_{1}, v_{2}$, and $w$. For better visibility, all the interactions, except for $u_{\|}$, are multiplied by 10 .

respectively [Fig. 1(b)]. $U(=\infty)$ is the on-site repulsion that enforces the hard-core constraint.

Figure 2 shows the field dependence of the parameters of $\mathcal{H}_{\text {eff }}$ in the field range of $\mu_{0} H=10-40 \mathrm{~T}$ where the energy scale of $\mu$ is larger than those of the other parameters by one order of magnitude. While all the hopping amplitudes have a strong field dependence, the relative change of the dominant attractive interaction $u_{\|}$remains very small over the whole field range. In other words, the ratio of the attractive interaction and the kinetic energy is widely tunable by the external field. This behavior resembles the case of ultracold atomic gases trapped in a periodic optical lattice, where the hopping amplitude is controlled by tuning the depth of the periodic potential [33]. In our case, however, the strong field dependence of $t_{\|}$and $t_{\perp}$ is caused by a different mechanism: The amplitude of the magnon tunneling via the "high-energy" sublattices 2 or 3 is inversely proportional to the energy barrier $\mu_{1}-\mu_{2}$. 
While the hopping amplitudes are comparable to each other, the interactions $u_{\|}^{\prime}, v_{2}$, and $w$ are much smaller than the other interactions in the field range of $\mu_{0} H=10-40 \mathrm{~T}$. We will then ignore these three interactions hereafter to reduce the computational cost of solving the two-body and three-body problems. Our exact diagonalization results for $\mathcal{H}_{\text {eff }}$ on a cluster of linear size $L=12$ (i.e., $2 \times L^{3}$ sites) confirm that these interactions have indeed a negligible effect.

\section{B. Numerical calculation methods for two- and three-magnon problems}

We numerically analyze the two- and three-magnon problems using the effective hard-core boson model, in which the number of magnons is conserved. The eigenvalues and the eigenstates of the two- and three-magnon sectors of the Hamiltonian (3) are obtained from exact diagonalization on finite lattices and from a numerical solution of the LippmannSchwinger equation. In the exact diagonalization method, we use the Krylov-Shur algorithm (library SLEPc [34]) to compute the lowest-energy state in each sector. The advantage of the exact diagonalization method is its simpler implementation. The calculations are performed with lattices of linear size $L \leqslant 72$ and $L \leqslant 18$ for the two- and three-magnon bound states, respectively. It is confirmed that these linear sizes are large enough to get accurate estimates of the $s$-wave scattering length and the binding energies of the two-magnon bound state and the lowest three-magnon bound state. The binding energy of the latter is well converged with respect to the system size, because its linear size is as small as a few lattice spacings. On the other hand, the same is not true for the first excited three-magnon bound state, because its linear size is larger and comparable to the maximum system size that can be reached with the state-of-the-art exact diagonalization method. However, its binding energy can still be computed by solving the Lippmann-Schwinger equation with the Gaussian quadrature rule for the numerical integrations in momentum space.

For the solution of the Lippmann-Schwinger equation, we consider essentially the same linear integral equations that were introduced in Ref. [27] for the case of the simple cubic lattice. However, the number of equations increases from 2 to 24 because of the multiple sublattice structure of the pyrochlore lattice. Detailed derivations of the LippmannSchwinger equations for the two- and three-magnon sectors are given in Appendix D.

\section{RESULTS}

\section{A. Single-magnon spectrum}

The single-magnon dispersion is obtained by diagonalizing the one-body component (first five terms) of $\mathcal{H}_{\text {eff }}$ (Appendix D2). For $t_{\|}, t^{\prime}, t_{\perp}<0$, the lower branch of the spectrum $E_{-}(\mathbf{k})$ has a minimum at $\mathbf{k}=\mathbf{0}$ where $\mathbf{k}=$ $\left(k_{1} \mathbf{G}_{1}+k_{2} \mathbf{G}_{2}+k_{3} \mathbf{G}_{3}\right) / 2 \pi$ with the reciprocal lattice vectors $\mathbf{G}_{1-3}$ for the primitive vectors $\mathbf{a}_{1-3}$ shown in Fig. 1(a). In the long-wavelength limit, $|\mathbf{k}| \ll 1$, we find

$$
E_{-}(\mathbf{k}) \simeq-\mu+2 t_{\|}+2 t_{\|}^{\prime}+8 t_{\perp}+\frac{\overline{\mathbf{k}}^{2}}{2 m_{z}},
$$

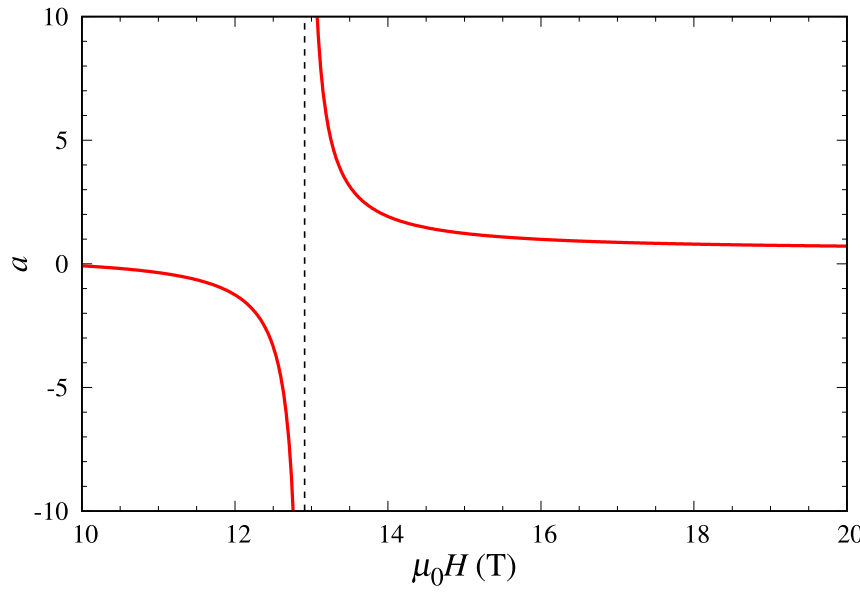

FIG. 3. $s$-wave scattering length in the unit of lattice spacing. The scattering length $a$ (red line) shows the divergent behavior of $a \propto\left(H-H_{c}\right)^{-1}$ near the two-magnon resonance condition $\mu_{0} H_{c}=$ $12.91 \mathrm{~T}$ (dashed line).

where $\quad \overline{\mathbf{k}}=\left[\sqrt{m_{z} / m_{x}}\left(k_{1}-k_{3} / 2\right), \sqrt{m_{z} / m_{x}}\left(k_{2}-k_{3} / 2\right), k_{3}\right]$, $m_{z}^{-1}=-t_{\|} / 2-2 t_{\|}^{\prime}-t_{\perp}$, and $m_{x}^{-1}=-4 t_{\perp}$. The effective masses $m_{z}$ and $m_{x}$ correspond to the [110] and the $\mathbf{G}_{1,2}(\perp[110])$ directions, respectively. Therefore, the low-energy physics of $\mathrm{Yb}_{2} \mathrm{Ti}_{2} \mathrm{O}_{7}$ is described by bosons in continuous space with anisotropic mass tensor. The two- and three-magnon binding energies discussed below are measured from the bottoms of two- and three-magnon continua at $E=2 E_{-}(\mathbf{0})$ and $3 E_{-}(\mathbf{0})$, respectively.

\section{B. Two-magnon resonance}

The low-energy scattering of magnons is parametrized by the $s$-wave scattering length $a$, which can be extracted from the solution of the two-magnon problem (Appendix D3). Because of the field dependence of the parameters of $\mathcal{H}_{\text {eff }}$, $a$ also varies with the magnetic field. Figure 3 shows the field dependence of $a$, establishing its magnetic field induced tunability. In particular, we find the divergent behavior of $a$ at $\mu_{0} H_{c}=12.91 \mathrm{~T}$, which corresponds to the two-magnon resonance condition and signals the onset of a two-magnon bound state for $H>H_{c}$. The green line in Fig. 4 then shows the field dependence of the two-magnon binding energy that is obtained from the exact diagonalization of $\mathcal{H}_{\text {eff }}$. As expected from the universality, the binding energy vanishes as $1 /\left(m_{z} a^{2}\right)$ upon approaching $H_{c}$. We note that the two-magnon boundstate dispersion has a global minimum at center-of-mass momentum $\mathbf{K}=\mathbf{0}$.

\section{Three-magnon Efimov states}

The exact diagonalization method is also applied to the three-magnon problem to compute the binding energies of the three-magnon bound states. Since the two-magnon bound state emerges for $H>H_{c}$, the lower threshold of the continuum is set by an eigenstate consisting of a two-magnon bound state or "bimagnon" plus a single magnon. Out of the few three-magnon bound states that appear below this threshold, we can identify two branches of $s$-wave bound states, labeled 


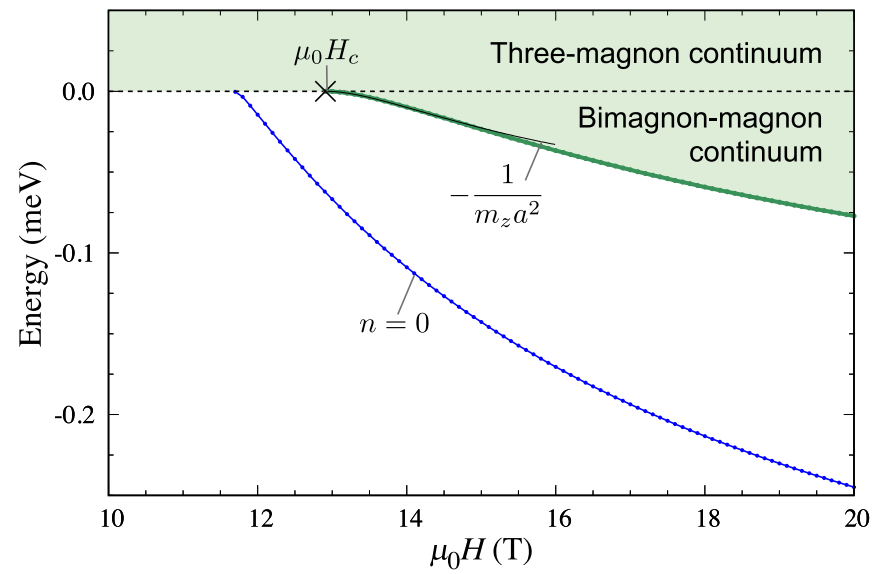

FIG. 4. Energy spectrum of the three-magnon states measured from $3 E_{-}(\mathbf{0})$. The cross $(\times)$ marks the critical field $\mu_{0} H_{c}$ for the twomagnon resonance. The green line represents the lower threshold of the bimagnon-magnon continuum corresponding to the negative of the two-magnon binding energy. The black thin line indicates the universal form of the two-magnon binding energy, $-1 /\left(m_{z} a^{2}\right)$, for $H \rightarrow H_{c}$. The blue circles represent the energy of the lowest $(n=0)$ three-magnon bound state corresponding to the negative of three-magnon binding energy.

by $n=0$ and $n=1$, as well as a branch of $p$-wave bound states. The $s$-wave bound states are candidates to be Efimov states. The binding energy of the lowest $(n=0)$ three-magnon bound state is shown in Fig. 4. The binding energy of the $n=$ 1 state is not shown there because it is too shallow and hard to distinguish from the two-magnon binding energy (green line).

We then focus on the $s$-wave three-magnon bound states ( $n=0$ and $n=1$ ) at the resonance condition $\mu_{0} H=12.91 \mathrm{~T}$. Our numerical solutions of the Lippmann-Schwinger equation (Appendix D4) produce well-converged binding energies for the $n=0$ and 1 states,

$$
\begin{aligned}
& E_{0}=0.062493 \mathrm{meV}, \\
& E_{1}=0.000180 \mathrm{meV},
\end{aligned}
$$

and the square root of their ratio is

$$
\sqrt{\frac{E_{0}}{E_{1}}}=18.6 .
$$

This value deviates from the universal value $\lambda=22.6944$, for $n \rightarrow \infty$, because the linear size of the $n=0$ state is comparable to the lattice spacing and lattice effects introduce a significant correction to its universal character. Similar deviations have been reported for the ratio $E_{0} / E_{1}$ obtained with a simpler spin Hamiltonian on a cubic lattice [27], where the ratios $E_{n} / E_{n+1}$ for $n=1,2$ are also computed and found to follow the universal value. Due to numerical limitations, we only have access to the $n=0$ and $n=1$ states. Consequently, to identify the Efimov character of each $n$ state, we are led to compare its wave function with the universal wave function of the Efimov state.

It is convenient to express the three-magnon wave function in a mixed representation, $\psi_{\mathbf{m}}(\mathbf{r} ; \mathbf{k})$, where $\mathbf{r}$ is the relative coordinate of two magnons and $\mathbf{k}$ is the relative momentum of the third magnon with respect to the center of mass of the other two. $\mathbf{m} \equiv\left(m_{1}, m_{2}, m_{3}\right)$ and $m_{j}=+,-$ corresponding to $\alpha=4,1$, respectively, identify the sublattice in which the $j$ th magnon resides. The center-of-mass momentum is set to zero because we are interested in the universal behavior that emerges in the long-wavelength limit of the theory. Similarly to the case of two-body bound states, the three-body bound states are expected to have minimum energy for center-ofmass momentum $\mathbf{K}=\mathbf{0}$ because the single-magnon spectrum has a global minimum at $\mathbf{k}=\mathbf{0}$ (Sec. III A). The threemagnon wave function is obtained by solving the LippmannSchwinger equation (Appendix D4). To compare the resulting wave functions against the universal theory, we express them in terms of the rescaled wave vector $\overline{\mathbf{k}}$ introduced in Eq. (4). Here, the effective masses of $m_{z}^{-1}=0.0399 \mathrm{meV}$ and $m_{x}^{-1}=$ $0.0425 \mathrm{meV}$ for $\mu_{0} H=12.91 \mathrm{~T}$ are used.

The wave functions of Efimov states obey

$$
\begin{gathered}
{\left[\frac{3}{4}\left(\frac{|\overline{\mathbf{k}}|}{\kappa_{n}}\right)^{2}+1\right] \psi_{\mathbf{m}}(\mathbf{r} ; \mathbf{k})=f\left(\sqrt{\frac{3}{4}} \frac{|\overline{\mathbf{k}}|}{\kappa_{n}}\right),} \\
f(z)=\frac{\sin \left[s_{0} \operatorname{arcsinh}(z)\right]}{s_{0} z} \sqrt{z^{2}+1},
\end{gathered}
$$

for $|\mathbf{r}| \lesssim 1$ and $|\overline{\mathbf{k}}| \ll 1$ (Appendix E). Here, the lattice spacing is adopted as the unit of length, $f(z)$ with $s_{0}=1.00624$ is the universal function [35], and $\kappa_{n}^{-1}=1 / \sqrt{m_{z} E_{n}}$ is a characteristic linear size of the $n$th bound state,

$$
\kappa_{0}^{-1}=0.799, \quad \kappa_{1}^{-1}=14.9 .
$$

The wave function in Eq. (8) is normalized to satisfy $\psi_{\mathbf{m}}\left(\mathbf{r} ; \mathbf{k}_{\text {min }}\right)=f(0)=1$, where $\left|\mathbf{k}_{\min }\right| \approx \mathbf{0}$ is the smallest wave number. The Efimov character of each state can be quantified by comparing the expression on the left-hand side of Eq. (8) against the universal function. The comparison shown in Fig. 5 reveals that the wave function of the $n=0$ exhibits some deviations from the universal behavior because of the above-mentioned lattice effect. However, the excellent agreement that is obtained for the $n=1$ state at long wavelengths $(|\overline{\mathbf{k}}|<1)$ confirms that these two states are indeed the bottom of the Efimov tower.

\section{SUMMARY AND DISCUSSIONS}

In this paper, we predict that the magnetic field acts as a knob to tune the $s$-wave scattering length of magnons in $\mathrm{Yb}_{2} \mathrm{Ti}_{2} \mathrm{O}_{7}$. Thus, the field plays the same role as in the Feshbach resonances of ultracold atomic gases. A twomagnon resonance condition is achieved at an experimentally reachable magnetic field strength of $\sim 13 \mathrm{~T}$ along the [110] direction, where the scattering length diverges and the binding transition occurs. As in the case of atomic gases, Efimov states are expected to emerge near this field value. Indeed, our calculations reveal a couple of three-magnon bound states with the $s$-wave wave function just below the three-magnon continuum of the excitation spectrum. While the ground state $(n=0)$ exhibits some deviations from the universal character due to lattice effects, the first excited state $(n=1)$ is indeed an Efimov state.

The results presented in this work are based on the Hamiltonian parameters of $\mathrm{Yb}_{2} \mathrm{Ti}_{2} \mathrm{O}_{7}$ reported in Ref. [31]. Other 
(a)

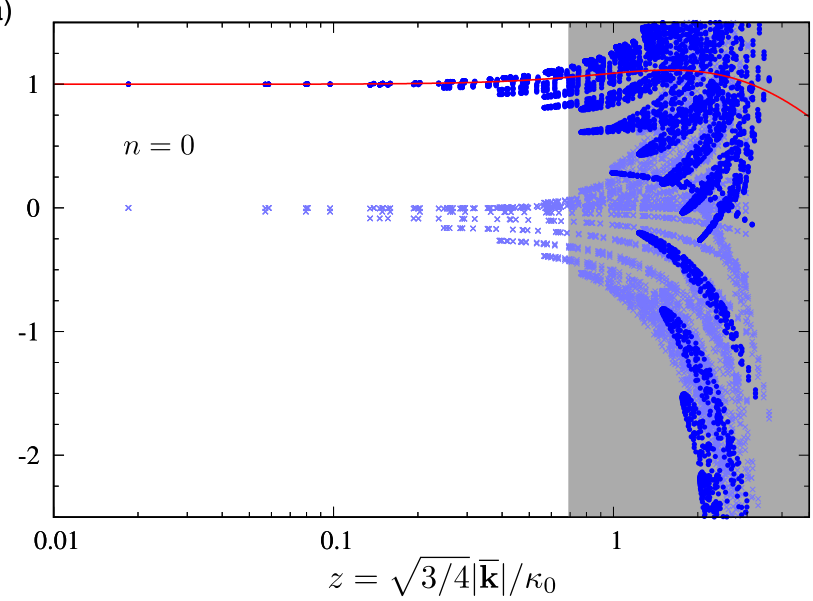

(b)

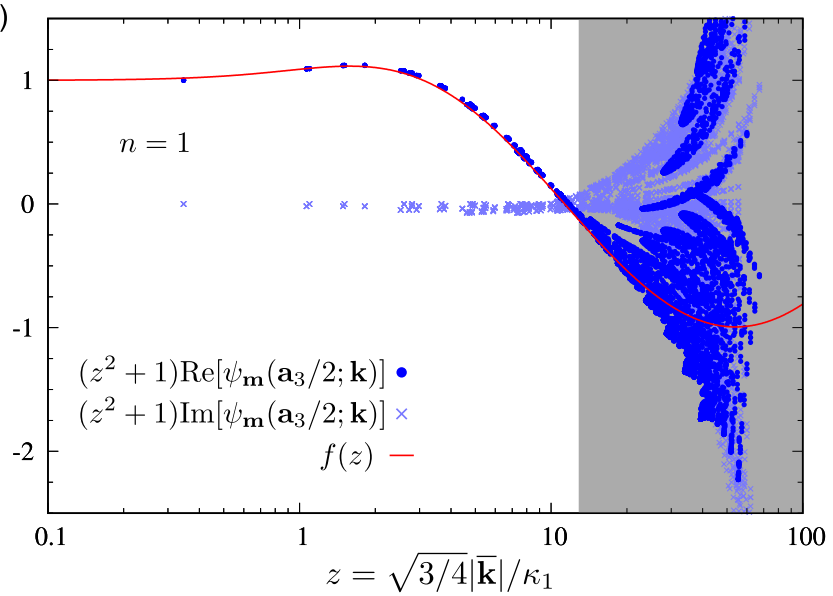

FIG. 5. Wave functions of the two lowest three-magnon bound states at the critical field $\mu_{0} H_{c}=12.91 \mathrm{~T}$. The universal function $f(z)$ and the left-hand side of Eq. (8) for (a) the ground state $(n=0)$ and for (b) the first excited state $(n=1)$ are compared as functions of the rescaled wave number $z=\sqrt{3 / 4}|\overline{\mathbf{k}}| / \kappa_{n}$ normalized by $\kappa_{n}$ for each $n$. Here, we set the relative coordinate as $\mathbf{r}=\mathbf{a}_{3} / 2$ so that a pair of particles occupy two nearest-neighbor sites. The gray shaded regions indicate the nonuniversal regime $(|\overline{\mathbf{k}}|>1)$.

experimental works [36-40] report larger values of $J_{z z}$, while the other parameters are almost the same. The resulting critical fields for the two-magnon resonance condition are

$$
\begin{aligned}
\text { Ref. [36] }: \mu_{0} H_{c} & =9.09 \mathrm{~T}, \\
\text { Refs. [37,38] }: \mu_{0} H_{c} & =8.80 \mathrm{~T}, \\
\text { Ref. [39] }: \mu_{0} H_{c} & =11.81 \mathrm{~T}, \\
\text { Ref. [40] }: \mu_{0} H_{c} & =11.48 \mathrm{~T} .
\end{aligned}
$$

$\mathrm{Yb}_{2} \mathrm{Ge}_{2} \mathrm{O}_{7}$ is another candidate material, whose Hamiltonian parameters have been recently reported [41]. In this case, the two-magnon resonance is achieved at

$$
\text { Ref. [41] }: \mu_{0} H_{c}=14.80 \mathrm{~T} \text {. }
$$

In all cases, the two-magnon resonance condition is achieved for experimentally reachable magnetic field values along the [110] direction.
Raman scattering is the ideal technique for detecting twomagnon bound states $[42,43]$. The effective Raman operator is a linear combination of the exchange interaction terms of the spin Hamiltonian $\tilde{\mathcal{H}}_{\text {spin }}$ (see Appendix B) [43]. After performing the canonical (unitary) transformation that transforms $\tilde{\mathcal{H}}_{\text {spin }}$ into $\mathcal{H}_{\text {eff }}$, the resulting effective Raman operator includes terms that create pairs of bosons. These terms are responsible for the transitions between the ground state and two-magnon bound states. Moreover, the effective Raman operator also includes terms that create three bosons, implying that three-magnon bound states can also be detected via Raman spectroscopy. These three-magnon terms of the effective Raman operator originate from the simultaneous presence of $a_{\mathbf{r}}^{\dagger} a_{\mathbf{r}^{\prime}}^{\dagger}$ and $n_{\mathbf{r}} a_{\mathbf{r}^{\prime}}^{\dagger}$ terms in the original hard-core boson model $\tilde{\mathcal{H}}_{\text {spin }}$. However, the intensity of the three-magnon absorption is smaller than the one for the two-magnon bound states by a factor of order $(J / H)^{2}$, where $J$ represents the energy scale of $J_{z \pm}, J_{ \pm \pm}$, or $J_{ \pm}$. The binding energy of the $n=0$ Efimov state is of order $0.1 \mathrm{meV}$ [Eq. (5)], implying that the required energy resolution is compatible with state-of-the-art THz Raman scattering [44]. The same is true for the binding energy of the two-magnon bound state (green thick line in Fig. 4). The binding energy of the $n=1$ state, on the other hand, is too small (of order $0.1 \mu \mathrm{eV}$ ) to be detected by the existing spectroscopic techniques. Neutron scattering can also be used to observe the two-magnon and three-magnon bound states $[45,46]$. However, the corresponding intensity is weak in both cases because it arises from the small hybridization of these bound states with the single-magnon state.

\section{ACKNOWLEDGMENTS}

The authors thank Y. Motome and R. Coldea for fruitful discussions. This work was partially supported by Japan Society for the Promotion of Science (JSPS) KAKENHI Grants No. JP15K17727, No. JP15H05855, No. 16H02206, and No. 18K03447 and JST, CREST Grant No. JPMJCR18T2, Japan. S.-S.Z. and C.D.B. are supported by funding from the Lincoln Chair of Excellence in Physics. Part of this work was carried out under the auspices of the U.S. DOE NNSA under Contract No. 89233218CNA000001 through the LDRD Program. This research used resources of the Oak Ridge Leadership Computing Facility at the Oak Ridge National Laboratory, which is supported by the Office of Science of the U.S. Department of Energy under Contract No. DE-AC05-00OR22725.

\section{APPENDIX A: SPIN HAMILTONIAN}

In this Appendix, we specify the local spin axes and the phase factors of the spin Hamiltonian $\mathcal{H}_{\text {spin }}$. The spin operators are expressed in a local reference frame whose $z$ axis is parallel to the local [111] direction. Following the notation of Ref. [31], the basis of the local reference frame for sublattice $\alpha=1-4$ reads

$$
\begin{array}{ll}
\mathbf{x}_{1}=\left(-\frac{2}{\sqrt{6}}, \frac{1}{\sqrt{6}}, \frac{1}{\sqrt{6}}\right), & \mathbf{z}_{1}=\left(\frac{1}{\sqrt{3}}, \frac{1}{\sqrt{3}}, \frac{1}{\sqrt{3}}\right), \\
\mathbf{x}_{2}=\left(-\frac{2}{\sqrt{6}},-\frac{1}{\sqrt{6}},-\frac{1}{\sqrt{6}}\right), & \mathbf{z}_{2}=\left(\frac{1}{\sqrt{3}},-\frac{1}{\sqrt{3}},-\frac{1}{\sqrt{3}}\right),
\end{array}
$$


$\mathbf{x}_{3}=\left(\frac{2}{\sqrt{6}}, \frac{1}{\sqrt{6}},-\frac{1}{\sqrt{6}}\right), \quad \mathbf{z}_{3}=\left(-\frac{1}{\sqrt{3}}, \frac{1}{\sqrt{3}},-\frac{1}{\sqrt{3}}\right)$,

$\mathbf{x}_{4}=\left(\frac{2}{\sqrt{6}},-\frac{1}{\sqrt{6}}, \frac{1}{\sqrt{6}}\right), \quad \mathbf{z}_{4}=\left(-\frac{1}{\sqrt{3}},-\frac{1}{\sqrt{3}}, \frac{1}{\sqrt{3}}\right)$,

and $\mathbf{y}_{\alpha}=\mathbf{z}_{\alpha} \times \mathbf{x}_{\alpha}$. The $g$-tensor takes the diagonal form in the local reference frame,

$$
g=\left[\begin{array}{ccc}
g_{\perp} & 0 & 0 \\
0 & g_{\perp} & 0 \\
0 & 0 & g_{\|}
\end{array}\right] .
$$

The phase factors in $\mathcal{H}_{\text {spin }}$ are

$$
\zeta=\left[\begin{array}{cccc}
0 & -1 & e^{i \frac{\pi}{3}} & e^{-i \frac{\pi}{3}} \\
-1 & 0 & e^{-i \frac{\pi}{3}} & e^{i \frac{\pi}{3}} \\
e^{i \frac{\pi}{3}} & e^{-i \frac{\pi}{3}} & 0 & -1 \\
e^{-i \frac{\pi}{3}} & e^{i \frac{\pi}{3}} & -1 & 0
\end{array}\right], \quad \gamma=-\zeta^{*} .
$$

\section{APPENDIX B: HARD-CORE BOSON REPRESENTATION OF THE SPIN HAMILTONIAN}

In this Appendix, we express the spin Hamiltonian in a new local reference frame and then apply a MatsubaraMatsuda transformation [32] (exact mapping between spin$1 / 2$ operators and hard-core bosons). The new local reference frame, defined by the three axes $\left(\tilde{\mathbf{x}}_{\alpha}, \tilde{\mathbf{y}}_{\alpha}, \tilde{\mathbf{z}}_{\alpha}\right)$, is such that $\tilde{\mathbf{z}}_{\alpha}$ is parallel to the direction of local magnetic moments, $\mathbf{m}_{\alpha}$, that minimizes the classical limit of $\mathcal{H}_{\text {spin }}$. In other words, $\tilde{\mathbf{z}}_{\alpha}$ is parallel to the direction of the magnetic moment that is obtained from a mean-field decoupling of the exchange interaction in $\mathcal{H}_{\text {spin }}$. In the new reference frame, we map the spin- $1 / 2$ operators into creation and annihilation operators of hard-core bosons,

$$
\begin{aligned}
& \tilde{\mathrm{S}}_{\mathbf{r}}^{z}=\frac{1}{2}-n_{\mathbf{r}}, \\
& \tilde{\mathrm{S}}_{\mathbf{r}}^{+}=\tilde{\mathrm{S}}_{\mathbf{r}}^{x}+i \tilde{\mathrm{S}}_{\mathbf{r}}^{y}=a_{\mathbf{r}}, \\
& \tilde{\mathrm{S}}_{\mathbf{r}}^{-}=\tilde{\mathbf{S}}_{\mathbf{r}}^{x}-i \tilde{\mathbf{S}}_{\mathbf{r}}^{y}=a_{\mathbf{r}}^{\dagger},
\end{aligned}
$$

with $n_{\mathbf{r}}=a_{\mathbf{r}}^{\dagger} a_{\mathbf{r}}$. The hard-core condition, $\left(a_{\mathbf{r}}^{\dagger}\right)^{2}=0, \forall \mathbf{r}$, is necessary to keep the dimension of the local Hilbert space equal to 2. The original spin Hamiltonian [Eq. (2) in the main text] can then be reexpressed as a Hamiltonian for a gas of hard-core bosons, whose particle number is not conserved. Up to an irrelevant constant, we obtain

$$
\begin{aligned}
\tilde{\mathcal{H}}_{\text {spin }}= & \sum_{\left\langle\mathbf{r} \mathbf{r}^{\prime}\right\rangle}\left[\left(\mathrm{t}_{\alpha_{\mathbf{r}} \alpha_{\mathbf{r}^{\prime}}} a_{\mathbf{r}}^{\dagger} a_{\mathbf{r}^{\prime}}+\text { H.c. }\right)+\mathrm{V}_{\alpha_{\mathbf{r}} \alpha_{\mathbf{r}^{\prime}}} n_{\mathbf{r}} n_{\mathbf{r}^{\prime}}\right. \\
& +\left(\mathrm{A}_{\alpha_{\mathbf{r}} \alpha_{\mathbf{r}^{\prime}}}^{++} a_{\mathbf{r}}^{\dagger} a_{\mathbf{r}^{\prime}}^{\dagger}+\text { H.c. }\right) \\
& \left.+\left(\mathrm{A}_{\alpha_{\mathbf{r}} \alpha_{\mathbf{r}^{\prime}}}^{n+} n_{\mathbf{r}} a_{\mathbf{r}^{\prime}}^{\dagger}+\mathrm{A}_{\alpha_{\mathbf{r}} \alpha_{\mathbf{r}^{\prime}}}^{+n} a_{\mathbf{r}^{\prime}}^{\dagger} n_{\mathbf{r}^{\prime}}+\text { H.c. }\right)\right] \\
& -\sum_{\mathbf{r}} \mu_{\alpha_{\mathbf{r}}} n_{\mathbf{r}}+U \sum_{\mathbf{r}} n_{\mathbf{r}}\left(n_{\mathbf{r}}-1\right) .
\end{aligned}
$$

The on-site repulsion $U=\infty$ enforces the hard-core constraint, while the other model parameters $\mu_{\alpha}, \mathrm{t}_{\alpha \alpha^{\prime}}, \mathrm{V}_{\alpha \alpha^{\prime}}, \mathrm{A}_{\alpha \alpha^{\prime}}^{++}$, $\mathrm{A}_{\alpha \alpha^{\prime}}^{n+}$, and $\mathrm{A}_{\alpha \alpha^{\prime}}^{+n}$ depend on the external field $H$. We note that the choice $\tilde{\mathbf{z}}_{\alpha} \| \mathbf{m}_{\alpha}$ eliminates all the linear terms in $a_{\mathbf{r}}$ and $a_{\mathbf{r}}^{\dagger}$ from $\tilde{\mathcal{H}}_{\text {spin }}$.

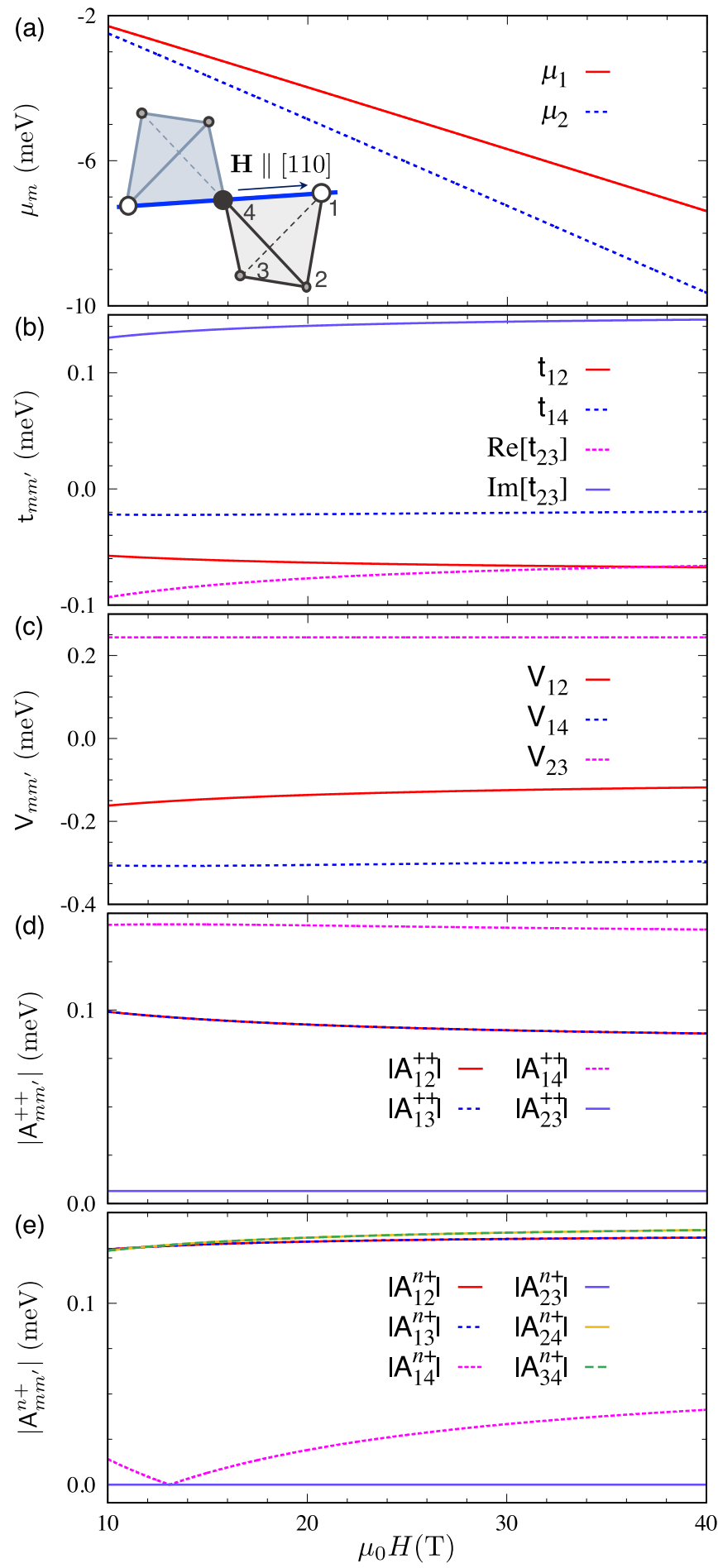

FIG. 6. Sublattice structure of pyrochlore lattice and model parameters for $\tilde{\mathcal{H}}_{\text {spin }}$. (a)-(e) Field dependence of the model parameters of $\tilde{\mathcal{H}}_{\text {spin }}$ obtained by applying a local rotation to the spin Hamiltonian $\mathcal{H}_{\text {spin }}$ of $\mathrm{Yb}_{2} \mathrm{Ti}_{2} \mathrm{O}_{7}$. Note that the following relation equations hold: $\mu_{1}=\mu_{4}, \mu_{2}=\mu_{3}, t_{12}=t_{13}=t_{24}=t_{34}, V_{12}=V_{13}=V_{24}=$ $\mathrm{V}_{34}, \mathrm{~A}_{12}^{++}=\mathrm{A}_{24}^{++}, \mathrm{A}_{13}^{++}=\mathrm{A}_{34}^{++}, \mathrm{A}_{12}^{n+}=-\mathrm{A}_{24}^{+n}, \mathrm{~A}_{13}^{n+}=-\mathrm{A}_{34}^{+n}, \mathrm{~A}_{14}^{n+}=$ $-\mathrm{A}_{14}^{+n}, \mathrm{~A}_{24}^{n+}=-\mathrm{A}_{12}^{+n}$, and $\mathrm{A}_{34}^{n+}=-\mathrm{A}_{13}^{+n}$. The inset of (a) shows a schematic view of the pyrochlore lattice with sublattice indices $1-4$.

Figure 6 shows the field dependence of the parameters of $\tilde{\mathcal{H}}_{\text {spin }}$ that are obtained for the exchange matrix and the $g$-tensor that have been reported for $\mathrm{Yb}_{2} \mathrm{Ti}_{2} \mathrm{O}_{7}$ [31]. Through a 


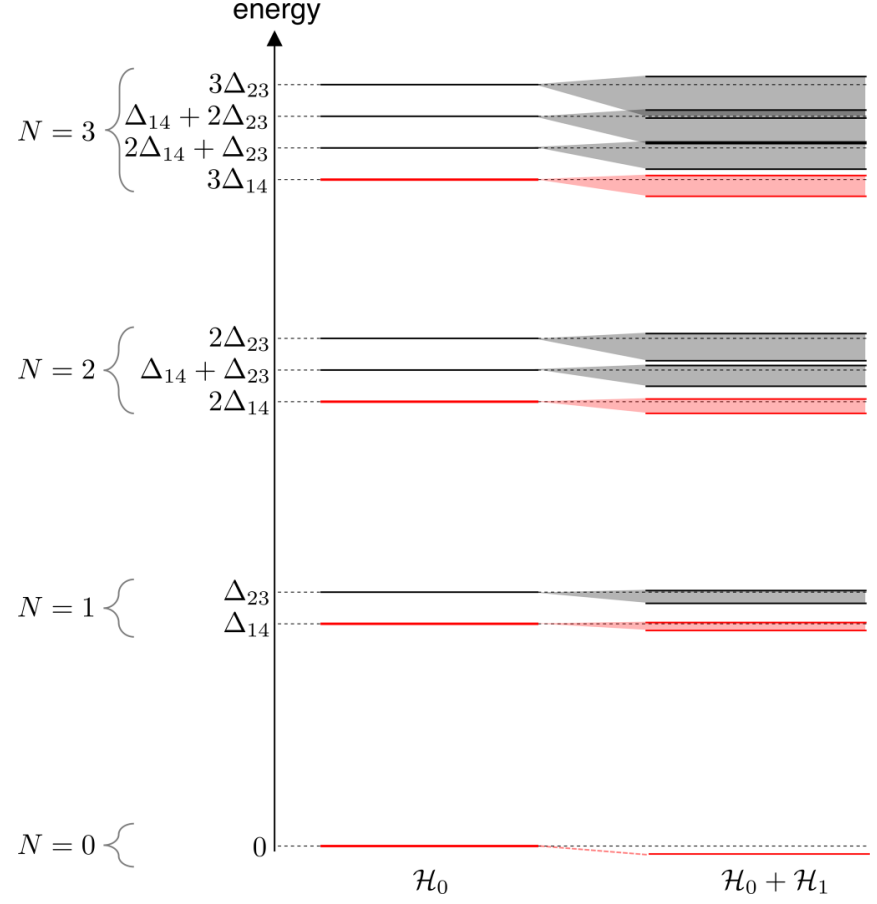

FIG. 7. Schematic view of energy spectrum of the unperturbed Hamiltonian $\mathcal{H}_{0}$ and the full Hamiltonian $\mathcal{H}_{0}+\mathcal{H}_{1}$ in the $N$-magnon sector with $N=0-3$. The red levels correspond to the states where all magnons occupy the low-energy sublattices 1 or 4 .

proper $U(1)$ gauge transformation, $a_{\mathbf{r}} \rightarrow a_{\mathbf{r}} e^{i \theta_{\mathbf{r}}}$, it is possible to make all the parameters $t_{\alpha_{\mathbf{r}} \alpha_{\mathbf{r}^{\prime}}}$ real, except for $t_{23}$, and, in addition, $\mathrm{t}_{12}=\mathrm{t}_{42}=\mathrm{t}_{31}=\mathrm{t}_{34}<0$ and $\mathrm{t}_{14}<0$.

\section{APPENDIX C: DERIVATION OF THE EFFECTIVE BOSON MODEL}

The effective Hamiltonian $\mathcal{H}_{\text {eff }}$ is derived from $\tilde{\mathcal{H}}_{\text {spin }}$ in the high-field regime by applying the second-order perturbation theory. For a strong enough field $H$, the chemical potential term becomes the dominant energy scale. We then divide $\tilde{\mathcal{H}}_{\text {spin }}$ into two parts,

$$
\begin{aligned}
\tilde{\mathcal{H}}_{\text {spin }}= & \mathcal{H}_{0}+\mathcal{H}_{1}, \\
\mathcal{H}_{0}= & -\sum_{\mathbf{r}} \mu_{\alpha_{\mathbf{r}}} n_{\mathbf{r}}+\mathrm{U} \sum_{\mathbf{r}} n_{\mathbf{r}}\left(n_{\mathbf{r}}-1\right), \\
\mathcal{H}_{1}= & \sum_{\left\langle\mathbf{r r}^{\prime}\right\rangle}\left[\left(\mathrm{t}_{\alpha_{\mathbf{r}} \alpha_{\mathbf{r}^{\prime}}} a_{\mathbf{r}^{\dagger}}^{\dagger} a_{\mathbf{r}^{\prime}}+\text { H.c. }\right)+\mathrm{V}_{\alpha_{\mathbf{r}} \alpha_{\mathbf{r}^{\prime}}} n_{\mathbf{r}} n_{\mathbf{r}^{\prime}}\right. \\
& +\left(\mathrm{A}_{\alpha_{\mathbf{r}} \alpha_{\mathbf{r}^{\prime}}}^{++} a_{\mathbf{r}^{\dagger}}^{\dagger} a_{\mathbf{r}^{\prime}}^{\dagger}+\text { H.c. }\right) \\
& \left.+\left(\mathrm{A}_{\alpha_{\mathbf{r}} \alpha_{\mathbf{r}^{\prime}}}^{n+} n_{\mathbf{r}^{\prime}} a_{\mathbf{r}^{\prime}}^{\dagger}+\mathrm{A}_{\alpha_{\mathbf{r}} \alpha_{\mathbf{r}^{\prime}}}^{+n} a_{\mathbf{r}^{\prime}}^{\dagger} n_{\mathbf{r}^{\prime}}+\text { H.c. }\right)\right],
\end{aligned}
$$

where $\mathcal{H}_{0}$ is the unperturbed part and $\mathcal{H}_{1}$ is the perturbation. The energy spectrum of the unperturbed Hamiltonian has discrete energy levels $E\left(N_{14}, E_{23}\right)=\Delta_{14} N_{14}+\Delta_{23} N_{23}$, where $\Delta_{\alpha} \equiv-\mu_{\alpha}, \Delta_{14}=\Delta_{1}=\Delta_{4}, \Delta_{23}=\Delta_{2}=\Delta_{3}$, and $N_{14}\left(N_{23}\right)$ is the number of hard-core bosons in sublattices 1 and 4 (2 and 3) (Fig. 7). $\mathcal{H}_{0}$ is massively degenerate in each sector $\left(N_{14}, N_{23}\right)$ except for $\left(N_{14}, N_{23}\right)=(0,0)$. The eigenvalues for different sectors are separated by an energy gap proportional to the field strength $H$. In the high-field regime, one can construct an effective Hamiltonian acting on each sector by treating $\mathcal{H}_{1}$ as a perturbation. For each degenerate subspace $\mathcal{S}_{E}$ of $\mathcal{H}_{0}$ with eigenenergy $E$, we introduce a projector $\mathcal{P}_{E}$ and the orthogonal projector $\mathcal{Q}_{E}=1-\mathcal{P}_{E}$. To second order in the perturbation, the effective Hamiltonian acting on $\mathcal{S}_{E}$ is given by

$$
\mathcal{H}_{\mathrm{eff}}(E)=E+\mathcal{P}_{E} \mathcal{H}_{1} \mathcal{P}_{E}+\mathcal{P}_{E} \mathcal{H}_{1} \mathcal{Q}_{E} \frac{1}{E-\mathcal{H}_{0}} \mathcal{Q}_{E} \mathcal{H}_{1} \mathcal{P}_{E}
$$

We are interested in the effective low-energy Hamiltonian that is obtained by projecting on the lowest-energy sector for each total number of magnons $N=N_{14}+N_{23}$. This is simply the sector that satisfies $N=N_{14}$ and $N_{23}=0$. For instance, let us consider the single-particle hopping amplitude $t_{\|}$between nearest-neighbor sites of the low-energy chains 1 or 4 [inset of Fig. 6(a)]. While the second term on the right-hand side of Eq. (C4) is simply $\mathrm{t}_{14} a_{4}^{\dagger} a_{1}$, the third term has contributions from multiple perturbation processes, including a numberconserving perturbation process where a magnon tunnels via sublattice 2 or sublattice 3 , e.g., hopping process $1 \rightarrow 2 \rightarrow 4$. By taking into account all the other perturbation processes, we obtain the nearest-neighbor intrachain hopping amplitude,

$$
\begin{aligned}
t_{\|}= & t_{14}-\frac{A_{12}^{++}\left(A_{24}^{++}\right)^{*}+A_{13}^{++}\left(A_{34}^{++}\right)^{*}}{\Delta_{14}+\Delta_{23}} \\
& -\frac{A_{14}^{n+}\left(A_{14}^{+n}\right)^{*}}{\Delta_{14}}-\frac{t_{12} t_{24}+t_{13} t_{34}}{\Delta_{23}-\Delta_{14}} .
\end{aligned}
$$

The other model parameters are obtained in a similar way,

$$
\begin{aligned}
t_{\|}^{\prime}= & -\frac{\left|\mathrm{A}_{14}^{++}\right|^{2}}{2 \Delta_{14}}, \\
t_{\perp}=t_{\perp}^{\prime}= & -\frac{\mathrm{A}_{12}^{++}\left(\mathrm{A}_{24}^{++}\right)^{*}}{\Delta_{23}+\Delta_{14}}-\frac{\mathrm{t}_{12} \mathrm{t}_{24}}{\Delta_{23}-\Delta_{14}} \\
= & -\frac{\mathrm{A}_{13}^{++}\left(\mathrm{A}_{34}^{++}\right)^{*}}{\Delta_{23}+\Delta_{14}}-\frac{\mathrm{t}_{13} \mathrm{t}_{34}}{\Delta_{23}-\Delta_{14}} \\
\mu= & \mu_{1}-2 \sum_{\gamma=2,3,4} \frac{\left|\mathrm{A}_{1 \gamma}^{++}\right|^{2}}{\Delta_{1}+\Delta_{\gamma}}+2 \sum_{\gamma=2,3} \frac{\mathrm{t}_{1 \gamma} \mathrm{t}_{\gamma 1}}{\Delta_{\gamma}-\Delta_{1}} \\
& +2 \sum_{\gamma=2,3,4} \frac{\mathrm{A}_{1 \gamma}^{n+}\left(\mathrm{A}_{1 \gamma}^{n+}\right)^{*}}{\Delta_{\gamma}}, \\
u_{\|}= & \mathrm{V}_{14}+\left[-\sum_{\gamma=2,3} \frac{\left(\mathrm{A}_{1 \gamma}^{n+}\right)^{*} \mathrm{~A}_{\gamma 4}^{+n}+\left(\mathrm{A}_{4 \gamma}^{n+}\right)^{*} \mathrm{~A}_{\gamma 1}^{+n}}{\Delta_{\gamma}}\right. \\
& +2 \frac{\left|\mathrm{A}_{41}^{n+}\right|^{2}+\left|\mathrm{A}_{14}^{n+}\right|^{2}}{\Delta_{14}}, \\
u_{\perp}= & -\frac{\left(\mathrm{A}_{12}^{n+}\right)^{*} \mathrm{~A}_{24}^{+n}+\mathrm{A}_{12}^{n+}\left(\mathrm{A}_{24}^{+n}\right)^{*}}{\Delta_{23}}-2 t_{\|}^{\prime}, \\
u_{\|}^{\prime}= & -2 \frac{\left(\mathrm{A}_{14}^{n+}\right)^{*} \mathrm{~A}_{41}^{+n}}{\Delta_{14}},
\end{aligned}
$$




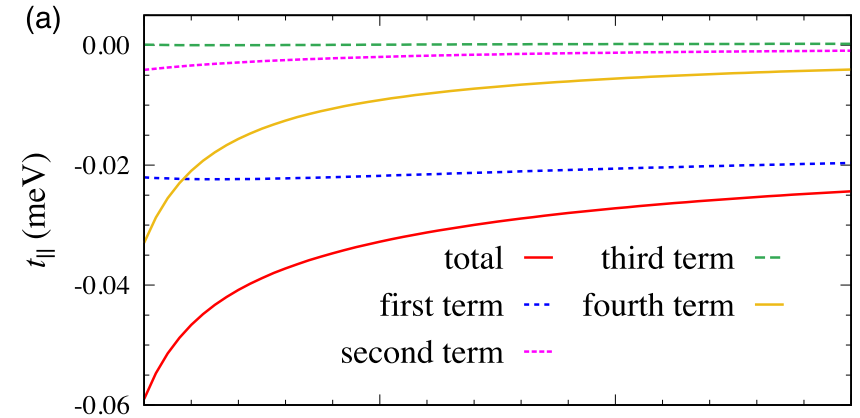

(b)

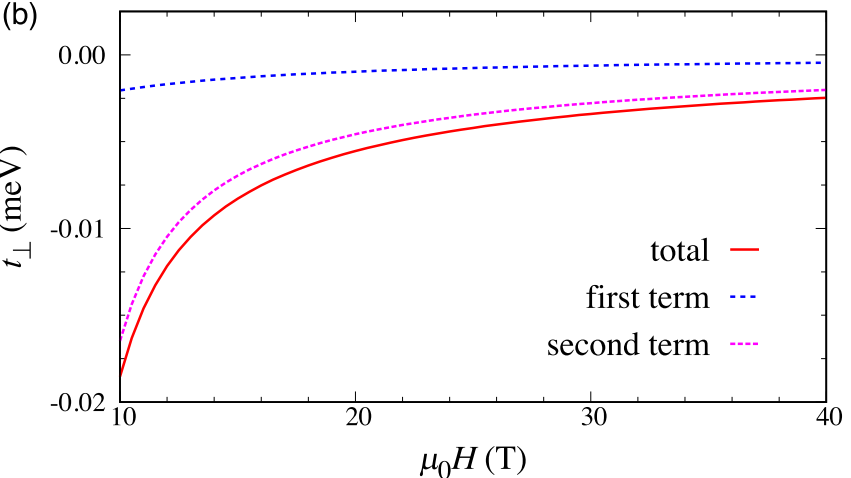

FIG. 8. Decomposition of the hopping amplitudes. (a) $t_{\|}$and (b) $t_{\perp}$ are decomposed into four and two terms as in the right-hand sides of Eqs. (C5) and (C7), respectively.

$$
\begin{aligned}
& v_{2}=-\frac{\left(\mathrm{A}_{14}^{n+}\right)^{*} \mathrm{~A}_{41}^{n+}}{\Delta_{14}}, \\
& w=2 \frac{\left|\mathrm{A}_{14}^{+n}\right|^{2}}{\Delta_{14}}-u_{\|}^{\prime} .
\end{aligned}
$$

The effective hopping amplitudes $t_{\|}$and $t_{\perp}$ have a strong field dependence because of the dominant tunneling processes via the high-energy chains 2 and 3 . Figure 8 shows the field dependence of each term on the right-hand sides of Eqs. (C5) and (C7), as well as the total values. The fourth term of Eq. (C5) and the second term of Eq. (C7) give the dominant contributions to the field dependence of the hopping amplitude. These terms correspond to the number-conserving perturbation processes discussed above.

In the main text, we focus on the resonance condition $\mu_{0} H=12.91 \mathrm{~T}$ where the model parameters (in $\mathrm{meV}$ ) are

$$
\begin{aligned}
t_{\|} & =-0.04357, \\
t_{\|}^{\prime} & =-0.003748, \\
t_{\perp}=t_{\perp}^{\prime} & =-0.01061, \\
\mu & =-2.736, \\
u_{\|} & =-0.2856, \\
u_{\|}^{\prime} & =-4.191 \times 10^{-7}, \\
u_{\perp} & =0.01090, \\
v_{1} & =0.007495, \\
v_{2} & =2.095 \times 10^{-7}, \\
w & =8.382 \times 10^{-7} .
\end{aligned}
$$

\section{APPENDIX D: SOLUTION OF THE FEW-BODY PROBLEM}

\section{Convenient notation of lattice site coordinates}

We first introduce a new notation for the lattice sites, which turns out to be convenient for implementing symmetry operations on the Lippmann-Schwinger equation. These operations are used to reduce the computational cost of solving the threemagnon problem. Each lattice site is specified by $\mathbf{r}=r_{x} \mathbf{e}_{x}+$ $r_{y} \mathbf{e}_{y}+r_{z} \mathbf{e}_{z}$ where $r_{x, y}=1,2, \ldots, L, r_{z}=1,2, \ldots, 2 L$, with $\mathbf{e}_{x}=\mathbf{a}_{1}-\mathbf{a}_{3} / 2, \mathbf{e}_{y}=\mathbf{a}_{2}-\mathbf{a}_{3} / 2$, and $\mathbf{e}_{z}=\mathbf{a}_{3} / 2$. The sign $m_{\mathbf{r}} \equiv(-1)^{r_{x}+r_{y}+r_{z}}$ is the sublattice index, namely $m_{\mathbf{r}}=+1$ $(-1)$ corresponds to the sublattice $\alpha_{\mathbf{r}}=4$ (1) (Fig. 1). The primitive translation vectors $\left(\mathbf{a}_{1,2,3}\right)$ and the reciprocal vectors $\left(\mathbf{G}_{1,2,3}\right)$ of the pyrochlore lattice are

$$
\begin{array}{ll}
\mathbf{a}_{1}=\sqrt{2} \ell(0,1,1), & \mathbf{G}_{1}=\frac{\pi}{\sqrt{2} \ell}(-1,1,1), \\
\mathbf{a}_{2}=\sqrt{2} \ell(1,0,1), \quad \mathbf{G}_{2}=\frac{\pi}{\sqrt{2} \ell}(1,-1,1), \\
\mathbf{a}_{3}=\sqrt{2} \ell(1,1,0), \quad \mathbf{G}_{3}=\frac{\pi}{\sqrt{2} \ell}(1,1,-1),
\end{array}
$$

where $\ell$ is the distance between nearest-neighbor $\mathrm{Yb}$ cation pairs. We take $\ell$ as the unit of length. For a finite lattice of $(2 \times$ $L^{3}$ ) sites with even $L$, the wave vectors in the first Brillouin zone are given by

$$
\mathbf{k}=\sum_{d=1}^{3} \mathbf{G}_{d} \frac{n_{d}}{L} \quad\left(n_{d}=-\frac{L}{2}, \ldots, \frac{L}{2}-1\right) .
$$

The summation over $\mathbf{k}$ becomes an integral in the infinite volume limit $L \rightarrow \infty$,

$$
\left.\frac{1}{L^{3}} \sum_{\mathbf{k}} \rightarrow \int_{\mathbf{k}} \equiv \iiint_{-\pi}^{\pi} \frac{d k_{1} d k_{2} d k_{3}}{(2 \pi)^{3}}\right|_{\mathbf{k}=\frac{1}{2 \pi} \sum_{d=1}^{3} k_{d} \mathbf{G}_{d}} .
$$

The integration over the first Brillouin zone is redefined by shifting the wave vector $\mathbf{k}$,

$$
\begin{aligned}
& \tilde{k}_{x}=k_{1}-\frac{k_{3}}{2} \\
& \tilde{k}_{y}=k_{2}-\frac{k_{3}}{2} \\
& \tilde{k}_{z}=k_{3}: \\
& \left.\int_{\mathbf{k}} \rightarrow \iiint_{-\pi}^{\pi} \frac{d \tilde{k}_{x} d \tilde{k}_{y} d \tilde{k}_{z}}{(2 \pi)^{3}}\right|_{\mathbf{k}=\frac{1}{2 \pi}\left(\tilde{k}_{x} \tilde{\mathbf{G}}_{x}+\tilde{\kappa}_{y} \tilde{\mathbf{G}}_{y}+\frac{\tilde{z}_{z}}{2} \tilde{\mathbf{G}}_{z}\right)},
\end{aligned}
$$

with

$$
\tilde{\mathbf{G}}_{x}=\mathbf{G}_{1}, \quad \tilde{\mathbf{G}}_{y}=\mathbf{G}_{2}, \quad \tilde{\mathbf{G}}_{z}=\mathbf{G}_{1}+\mathbf{G}_{2}+2 \mathbf{G}_{3} .
$$

We choose the subscript $\{x, y, z\}$ for $\tilde{\mathbf{G}}$ instead of $\{1,2,3\}$ because $\tilde{\mathbf{G}}_{x, y, z}$ can be regarded as the reciprocal vectors of the lattice spanned by the primitive vectors $\mathbf{e}_{x, y, z}$,

$$
2 \pi \frac{\tilde{\mathbf{G}}_{\mu} \times \tilde{\mathbf{G}}_{\nu}}{\tilde{\mathbf{G}}_{x} \cdot\left(\tilde{\mathbf{G}}_{y} \times \tilde{\mathbf{G}}_{z}\right)}=\sum_{\gamma} \epsilon_{\mu \nu \gamma} \mathbf{e}_{\gamma},
$$

where $\epsilon_{\mu \nu \gamma}$ is the Levi-Civita tensor.

In this notation, the effective boson Hamiltonian becomes

$$
\begin{aligned}
\mathcal{H}_{\mathrm{eff}}= & \sum_{\mathbf{r}}\left[\sum_{\mathbf{e}} t_{\mathbf{e}}^{m_{\mathbf{r}}} a_{\mathbf{r}}^{\dagger} a_{\mathbf{r}+\mathbf{e}}+\sum_{\mathbf{e}, \mathbf{e}^{\prime}} u_{\mathbf{e e}^{\prime}}^{m_{\mathbf{r}} m_{\mathbf{r}+\mathbf{e}}} a_{\mathbf{r}}^{\dagger} a_{\mathbf{r}+\mathbf{e}}^{\dagger} a_{\mathbf{r}+\mathbf{e}^{\prime}} a_{\mathbf{r}}\right] \\
& -\mu \sum_{\mathbf{r}} a_{\mathbf{r}}^{\dagger} a_{\mathbf{r}} .
\end{aligned}
$$


The hopping amplitudes $t_{\mathbf{e}}^{m}$ and the two-body interactions $u_{\mathbf{e e}^{\prime}}^{m m^{\prime}}$ are defined as

$$
\begin{gathered}
t_{\mathbf{e}}^{m}= \begin{cases}t_{\|} & \text {if } \mathbf{e}= \pm \mathbf{e}_{z}, \\
t_{\|}^{\prime} & \text { if } \mathbf{e}= \pm 2 \mathbf{e}_{z}, \\
t_{\perp} & \text { if } \mathbf{e}= \pm \mathbf{e}_{x, y}, \pm\left(\mathbf{e}_{x, y}+m \mathbf{e}_{z}\right), \\
0 & \text { otherwise, }\end{cases} \\
u_{\mathbf{e e}^{\prime}}^{m m^{\prime}}= \begin{cases}u_{\|} & \text {if } \mathbf{e}=\mathbf{e}^{\prime}=\mathbf{e}_{z}, m=\overline{m^{\prime}}, \\
u_{\perp} & \text { if } \mathbf{e}=\mathbf{e}^{\prime}=\mathbf{e}_{x, y}, m=\overline{m^{\prime}}, \\
-u_{\perp} & \text { if } \mathbf{e}=\mathbf{e}^{\prime}=\left(\mathbf{e}_{x, y}+m \mathbf{e}_{z}\right), m=m^{\prime}, \\
v_{1} & \text { if } \mathbf{e}=-\mathbf{e}^{\prime}= \pm \mathbf{e}_{z}, m=\overline{m^{\prime}}, \\
\mathbf{U}=\infty & \text { if } \mathbf{e}=\mathbf{e}^{\prime}=0, m=m^{\prime}, \\
0 & \text { otherwise },\end{cases}
\end{gathered}
$$

where $\bar{m} \equiv-m$. Note that we neglected $u_{\|}^{\prime}, v_{2}$, and $w$ because they are much smaller than the other interactions as discussed in the main text (Sec. II A).

In what follows, we consider the one-, two-, and threemagnon subspaces spanned by one-magnon states $a_{\mathbf{r}_{1}}^{\dagger}|\emptyset\rangle$, twomagnon states $a_{\mathbf{r}_{1}}^{\dagger} a_{\mathbf{r}_{2}}^{\dagger}|\emptyset\rangle$, and three-magnon states $a_{\mathbf{r}_{1}}^{\dagger} a_{\mathbf{r}_{2}}^{\dagger} a_{\mathbf{r}_{3}}^{\dagger}|\emptyset\rangle$, respectively. The boson vacuum $|\emptyset\rangle$ represents the ground state.

\section{Single-magnon problem}

The projection of the Schrödinger equation, $E|\Psi\rangle=$ $\mathcal{H}_{\text {eff }}|\Psi\rangle$, onto the single-magnon basis states $a_{\mathbf{r}}^{\dagger}|\emptyset\rangle$ leads to

$$
E \Psi(\mathbf{r})=\left\langle\emptyset\left|a_{\mathbf{r}} \mathcal{H}_{\mathrm{eff}}\right| \Psi\right\rangle=\sum_{\mathbf{e}} t_{\mathbf{e}}^{m_{\mathbf{r}}} \Psi(\mathbf{r}+\mathbf{e}),
$$

where $|\Psi\rangle$ is in the one-magnon subspace, and $\Psi(\mathbf{r})=$ $\left\langle\emptyset\left|a_{\mathbf{r}}\right| \Psi\right\rangle$. From the Fourier transform,

$$
\tilde{\Psi}_{m}(\mathbf{k})=\sum_{\mathbf{r} \in m} e^{-i \mathbf{k} \cdot \mathbf{r}} \Psi(\mathbf{r}), \quad \Psi(\mathbf{r})=\frac{1}{L^{3}} \sum_{\mathbf{k}} e^{i \mathbf{k} \cdot \mathbf{r}} \tilde{\Psi}_{m_{\mathbf{r}}}(\mathbf{k}),
$$

where the sum of $\mathbf{r} \in m$ runs over all the lattice sites of a given sublattice, $m_{\mathbf{r}}=m$, we obtain

$$
E\left[\begin{array}{c}
\tilde{\Psi}_{+}(\mathbf{k}) \\
\tilde{\Psi}_{-}(\mathbf{k})
\end{array}\right]=\left[\begin{array}{ll}
\varepsilon_{\mathbf{k}++} & \varepsilon_{\mathbf{k}+-} \\
\varepsilon_{\mathbf{k}-+} & \varepsilon_{\mathbf{k}--}
\end{array}\right]\left[\begin{array}{c}
\tilde{\Psi}_{+}(\mathbf{k}) \\
\tilde{\Psi}_{-}(\mathbf{k})
\end{array}\right],
$$

where

$$
\begin{aligned}
\varepsilon_{\mathbf{k} m m}= & -\mu+2 t_{\|}^{\prime} \cos \left(2 \mathbf{k} \cdot \mathbf{e}_{z}\right) \\
& +2 t_{\perp}\left[\cos \left(\mathbf{k} \cdot \mathbf{e}_{x}+m \mathbf{k} \cdot \mathbf{e}_{z}\right)+\cos \left(\mathbf{k} \cdot \mathbf{e}_{y}+m \mathbf{k} \cdot \mathbf{e}_{z}\right)\right], \\
\varepsilon_{\mathbf{k} m \bar{m}}= & 2 t_{\|} \cos \left(\mathbf{k} \cdot \mathbf{e}_{z}\right)+2 t_{\perp}\left[\cos \left(\mathbf{k} \cdot \mathbf{e}_{x}\right)+\cos \left(\mathbf{k} \cdot \mathbf{e}_{y}\right)\right] .
\end{aligned}
$$

The single-magnon spectrum is given by the eigenvalues of the matrix $\varepsilon_{\mathbf{k}}$.

\section{Two-magnon problem}

In this section, we explain how to compute the $s$-wave scattering length and the binding energy of the two-magnon bound state using the Lippmann-Schwinger equation. Similarly to the single-magnon problem, the projection of the Schrödinger equation, $E|\Psi\rangle=\mathcal{H}_{\text {eff }}|\Psi\rangle$, onto the (unnormalized) two-magnon basis states $a_{\mathbf{r}_{1}}^{\dagger} a_{\mathbf{r}_{2}}^{\dagger}|\emptyset\rangle$ leads to

$$
\begin{aligned}
E \Psi\left(\mathbf{r}_{1}, \mathbf{r}_{2}\right)= & \left\langle\emptyset\left|a_{\mathbf{r}_{2}} a_{\mathbf{r}_{1}} \mathcal{H}_{\mathrm{eff}}\right| \Psi\right\rangle \\
= & \sum_{\mathbf{e}}\left[t_{\mathbf{e}}^{m_{\mathbf{r}_{1}}} \Psi\left(\mathbf{r}_{1}+\mathbf{e}, \mathbf{r}_{2}\right)+t_{\mathbf{e}}^{m_{\mathbf{r}_{2}}} \Psi\left(\mathbf{r}_{1}, \mathbf{r}_{2}+\mathbf{e}\right)\right] \\
& +\sum_{\mathbf{e}, \mathbf{e}^{\prime}}\left[\delta_{\mathbf{r}_{2}} \mathbf{r}_{1}+\mathbf{e} u_{\mathbf{e e}^{\prime}}^{m_{\mathrm{r}_{1}} m_{\mathbf{r}_{1}+\mathbf{e}}} \Psi\left(\mathbf{r}_{1}, \mathbf{r}_{1}+\mathbf{e}^{\prime}\right)\right. \\
& \left.+\delta_{\mathbf{r}_{1} \mathbf{r}_{2}+\mathbf{e}} u_{\mathbf{e e}^{\prime}}^{m_{\mathbf{r}_{2}} m_{\mathbf{r}_{2}+\mathbf{e}}} \Psi\left(\mathbf{r}_{2}+\mathbf{e}^{\prime}, \mathbf{r}_{2}\right)\right],
\end{aligned}
$$

where $\Psi\left(\mathbf{r}_{1}, \mathbf{r}_{2}\right)=\left\langle\emptyset\left|a_{\mathbf{r}_{2}} a_{\mathbf{r}_{1}}\right| \Psi\right\rangle$. Here, we focus on the twomagnon scattering in the long-wavelength limit $(\mathbf{k} \rightarrow \mathbf{0})$ to compute the $s$-wave scattering length $a$. For this purpose, we consider the bottom of the two-magnon continuum with zero center-of-mass momentum $\mathbf{K}=\mathbf{0}$ and energy $E=2 \mathcal{E}_{0}\left(\mathcal{E}_{0}=\right.$ $\left.-\mu+2 t_{\|}+2 t_{\|}^{\prime}+8 t_{\perp}\right)$. From the Fourier transform,

$$
\tilde{\Psi}_{m_{1} m_{2}}\left(\mathbf{k}_{1}, \mathbf{k}_{2}\right)=\sum_{\mathbf{r}_{1} \in m_{1}, \mathbf{r}_{2} \in m_{2}} e^{-i\left(\mathbf{k}_{1} \cdot \mathbf{r}_{1}+\mathbf{k}_{2} \cdot \mathbf{r}_{2}\right)} \Psi\left(\mathbf{r}_{1}, \mathbf{r}_{2}\right),
$$

$$
\Psi\left(\mathbf{r}_{1}, \mathbf{r}_{2}\right)=\frac{1}{L^{6}} \sum_{\mathbf{k}_{1}, \mathbf{k}_{2}} e^{i\left(\mathbf{k}_{1} \cdot \mathbf{r}_{1}+\mathbf{k}_{2} \cdot \mathbf{r}_{2}\right)} \tilde{\Psi}_{m_{\mathbf{r}_{1}} m_{\mathbf{r}_{2}}}\left(\mathbf{k}_{1}, \mathbf{k}_{2}\right),
$$

we obtain

$$
\begin{aligned}
& \sum_{m_{1}^{\prime}, m_{2}^{\prime}}\left[E \delta_{m_{1} m_{1}^{\prime}} \delta_{m_{2} m_{2}^{\prime}}-\varepsilon_{\mathbf{k}_{1} m_{1} m_{1}^{\prime}} \delta_{m_{2} m_{2}^{\prime}}-\varepsilon_{\mathbf{k}_{2} m_{2} m_{2}^{\prime}} \delta_{m_{1} m_{1}^{\prime}}\right]\left[\tilde{\Psi}_{m_{1}^{\prime} m_{2}^{\prime}}\left(\mathbf{k}_{1}, \mathbf{k}_{2}\right)-\delta_{\mathbf{k}_{1} \mathbf{0}} \delta_{\mathbf{k}_{2} \mathbf{0}} \phi_{m_{1}^{\prime} m_{2}^{\prime}} L^{3}\right] \\
& \quad=\frac{1}{L^{3}} \sum_{\mathbf{k}_{2}^{\prime}} \sum_{\mathbf{e}, \mathbf{e}^{\prime}}\left[e^{-i\left(\mathbf{k}_{2} \cdot \mathbf{e}-\mathbf{k}_{2}^{\prime} \cdot \mathbf{e}^{\prime}\right)} u_{\mathbf{e e}^{\prime}}^{m_{1} m_{2}}+e^{-i\left\{\mathbf{k}_{1} \cdot \mathbf{e}-\left(\mathbf{k}_{1}+\mathbf{k}_{2}-\mathbf{k}_{2}^{\prime}\right) \cdot \mathbf{e}^{\prime}\right\}} u_{\mathbf{e}^{\prime}}^{m_{2} m_{1}}\right] \tilde{\Psi}_{m_{1} m_{2}}\left(\mathbf{k}_{1}+\mathbf{k}_{2}-\mathbf{k}_{2}^{\prime}, \mathbf{k}_{2}^{\prime}\right),
\end{aligned}
$$

where $m_{\mathbf{e}}=m_{\mathbf{e}^{\prime}}$ is always satisfied for finite $u_{\mathbf{e e}^{\prime}}^{m m^{\prime}}$. $\phi_{m_{1}^{\prime} m_{2}^{\prime}}$ represents the two-magnon eigenstate of the noninteracting problem with eigenvalue $2 \mathcal{E}_{0}$ in momentum space,

$$
\sum_{m_{1}^{\prime}, m_{2}^{\prime}}\left[\varepsilon_{\mathbf{0} m_{1} m_{1}^{\prime}} \delta_{m_{2} m_{2}^{\prime}}+\varepsilon_{\mathbf{0} m_{2} m_{2}^{\prime}} \delta_{m_{1} m_{1}^{\prime}}\right] \phi_{m_{1}^{\prime} m_{2}^{\prime}}=2 \mathcal{E}_{0} \phi_{m_{1} m_{2}},
$$

$$
\sum_{m_{1}^{\prime}, m_{2}^{\prime}} \phi_{m_{1}^{\prime} m_{2}^{\prime}}^{*} \phi_{m_{1}^{\prime} m_{2}^{\prime}}=1
$$

that has a simple solution, $\phi_{m_{1} m_{2}}=1 / 2 \forall\left(m_{1}, m_{2}\right)$, for $\mathbf{k}_{1}=\mathbf{k}_{2}=\mathbf{0}$. We only consider states with zero centerof-mass momentum and impose the ansatz $\tilde{\Psi}_{m m^{\prime}}\left(\mathbf{k}_{1}, \mathbf{k}_{2}\right)=$ $\delta_{\mathbf{k}_{1}-\mathbf{k}_{2}} \tilde{\psi}_{m m^{\prime}}\left(\mathbf{k}_{2}\right)$, which is symmetric under an exchange of 
two bosons: $\tilde{\psi}_{m m^{\prime}}(\mathbf{k})=\tilde{\psi}_{m^{\prime} m}(-\mathbf{k})$. The unknown functions $\tilde{\psi}_{m m^{\prime}}(\mathbf{k})$ satisfy the Lippmann-Schwinger equation,

$$
\begin{aligned}
\tilde{\psi}_{m_{1} m_{2}}(\mathbf{k})= & \delta_{\mathbf{k} \mathbf{0}} \phi_{m_{1} m_{2}} L^{3}+\frac{1}{L^{3}} \sum_{\mathbf{k}^{\prime}} \sum_{m_{1}^{\prime}, m_{2}^{\prime}} \sum_{\mathbf{e}, \mathbf{e}^{\prime}}[G(\mathbf{k})]_{m_{1} m_{2} ; m_{1}^{\prime} m_{2}^{\prime}} \\
& \times\left\{e^{-i\left(\mathbf{k} \cdot \mathbf{e}-\mathbf{k}^{\prime} \cdot \mathbf{e}^{\prime}\right)} u_{\mathbf{e e}^{\prime}}^{m_{1}^{\prime} m_{2}^{\prime}}+e^{i\left(\mathbf{k} \cdot \mathbf{e}-\mathbf{k}^{\prime} \cdot \mathbf{e}^{\prime}\right)} u_{\mathbf{e e}^{\prime}}^{m_{1}^{\prime} m_{2}^{\prime}}\right\} \\
& \times \tilde{\psi}_{m_{1}^{\prime} m_{2}^{\prime}}\left(\mathbf{k}^{\prime}\right),
\end{aligned}
$$

where the propagator matrix $G(\mathbf{k})$ is defined as

$$
\begin{aligned}
& {\left[G^{-1}(\mathbf{k})\right]_{m_{1} m_{2} ; m_{1}^{\prime} m_{2}^{\prime}}} \\
& \quad \equiv E \delta_{m_{1} m_{1}^{\prime}} \delta_{m_{2} m_{2}^{\prime}}-\varepsilon_{-\mathbf{k} m_{1} m_{1}^{\prime}} \delta_{m_{2} m_{2}^{\prime}}-\varepsilon_{\mathbf{k} m_{2} m_{2}^{\prime}} \delta_{m_{1} m_{1}^{\prime}} \\
& \quad=\left[G^{-1}(-\mathbf{k})\right]_{m_{2} m_{1} ; m_{2}^{\prime} m_{1}^{\prime}} .
\end{aligned}
$$

The last equality arises from the exchange symmetry of bosons. The inverse Fourier transform, $\psi_{m_{1} m_{2}}(\mathbf{r})=$ $\frac{1}{L^{3}} \sum_{\mathbf{k}} e^{i \mathbf{k} \cdot \mathbf{r}} \tilde{\psi}_{m_{1} m_{2}}(\mathbf{k})$, leads to

$$
\begin{aligned}
\psi_{m_{1} m_{2}}(\mathbf{r})= & \phi_{m_{1} m_{2}}+\int_{\mathbf{k}} \sum_{m_{1}^{\prime}, m_{2}^{\prime}} \sum_{\mathbf{e}, \mathbf{e}^{\prime}}\left[e^{i \mathbf{k} \cdot \mathbf{r}}[G(\mathbf{k})]_{m_{1} m_{2} ; m_{1}^{\prime} m_{2}^{\prime}}\right. \\
& \left.+e^{-i \mathbf{k} \cdot \mathbf{r}}[G(\mathbf{k})]_{m_{2} m_{1} ; m_{1}^{\prime} m_{2}^{\prime}}\right] e^{-i \mathbf{k} \cdot \mathbf{e}} u_{\mathbf{e e}^{\prime}}^{m_{1}^{\prime} m_{2}^{\prime}} \psi_{m_{1}^{\prime} m_{2}^{\prime}}\left(\mathbf{e}^{\prime}\right),
\end{aligned}
$$

in the infinite volume limit $L \rightarrow \infty$. Note that the properties $[G(\mathbf{k})]_{m_{1} m_{2} ; m_{1}^{\prime} m_{2}^{\prime}}=[G(-\mathbf{k})]_{m_{2} m_{1} ; m_{2}^{\prime} m_{1}^{\prime}}, u_{\mathbf{e e}^{\prime}}^{m_{1}^{\prime} m_{2}^{\prime}}=u_{\mathbf{e e}^{\prime}}^{m_{2}^{\prime} m_{1}^{\prime}}$, and $\psi_{m_{1}^{\prime} m_{2}^{\prime}}(\mathbf{r})=\psi_{m_{2}^{\prime} m_{1}^{\prime}}(-\mathbf{r})$ are used to derive Eq. (D22), which leads to a linear system of equations for the 12 unknown variables $\psi_{m_{1} m_{2}}(\mathbf{e})$. These 12 unknown variables and the interaction matrix elements acting on them are summarized as

$$
\begin{aligned}
\psi_{+-}\left(\mathbf{e}_{z}\right) & \Rightarrow u_{\mathbf{e}_{z} \mathbf{e}_{z}}^{+-}=u_{\|}, \quad u_{\left(-\mathbf{e}_{z}\right)\left(+\mathbf{e}_{z}\right)}^{+-}=v_{1}, \\
\psi_{-+}\left(\mathbf{e}_{z}\right) & \Rightarrow u_{\mathbf{e}_{z} \mathbf{e}_{z}}^{-+}=u_{\|}, \quad u_{\left(-\mathbf{e}_{z}\right)\left(+\mathbf{e}_{z}\right)}^{-+}=v_{1}, \\
\psi_{+-}\left(\mathbf{e}_{x, y}\right) & \Rightarrow u_{\mathbf{e}_{x, y}^{+-} \mathbf{e}_{x, y}}^{+}=u_{\perp}, \\
\psi_{-+}\left(\mathbf{e}_{x, y}\right) & \Rightarrow u_{\mathbf{e}_{x, y}}^{-+} \mathbf{e}_{x, y}=u_{\perp}, \\
\psi_{++}\left(\mathbf{e}_{x, y}+\mathbf{e}_{z}\right) & \Rightarrow u_{\left(\mathbf{e}_{x, y}+\mathbf{e}_{z}\right)\left(\mathbf{e}_{x, y}+\mathbf{e}_{z}\right)}^{++}=-u_{\perp}, \\
\psi_{--}\left(\mathbf{e}_{x, y}-\mathbf{e}_{z}\right) & \Rightarrow u_{\left(\mathbf{e}_{x, y}-\mathbf{e}_{z}\right)\left(\mathbf{e}_{x, y}-\mathbf{e}_{z}\right)}^{-}=-u_{\perp}, \\
\psi_{++}(\mathbf{0}) & \Rightarrow u_{\mathbf{0 0}}^{++}=\mathrm{U}=\infty, \\
\psi_{--}(\mathbf{0}) & \Rightarrow u_{\mathbf{0 0}}^{--}=\mathrm{U}=\infty .
\end{aligned}
$$

Note that $\psi_{m_{1} m_{2}}(\mathbf{r})=\psi_{m_{2} m_{1}}(-\mathbf{r})$ by the exchange symmetry of bosons, implying that $\psi_{+_{-}}\left(-\mathbf{e}_{z}\right)=\psi_{-+}\left(\mathbf{e}_{z}\right)$ and $\psi_{-+}\left(-\mathbf{e}_{z}\right)=\psi_{+-}\left(\mathbf{e}_{z}\right)$. For concreteness, Eq. (D22) can be expressed as

$$
\mathcal{I} \boldsymbol{\psi}=\boldsymbol{\phi}+\mathcal{A}(E) \boldsymbol{\psi} \Rightarrow \boldsymbol{\psi}=[\mathcal{I}-\mathcal{A}(E)]^{-1} \boldsymbol{\phi},
$$

with

$$
\begin{aligned}
\boldsymbol{\psi}^{\mathrm{t}}= & {\left[\psi_{+-}\left(\mathbf{e}_{z}\right), \psi_{-+}\left(\mathbf{e}_{z}\right), \psi_{+-}\left(\mathbf{e}_{x}\right), \psi_{+-}\left(\mathbf{e}_{y}\right),\right.} \\
& \psi_{-+}\left(\mathbf{e}_{x}\right), \psi_{-+}\left(\mathbf{e}_{y}\right), \psi_{++}\left(\mathbf{e}_{x}+\mathbf{e}_{z}\right), \psi_{++}\left(\mathbf{e}_{y}+\mathbf{e}_{z}\right), \\
& \left.\psi_{--}\left(\mathbf{e}_{x}-\mathbf{e}_{z}\right), \psi_{--}\left(\mathbf{e}_{y}-\mathbf{e}_{z}\right), \bigcup \psi_{++}(\mathbf{0}), \bigcup \psi_{--}(\mathbf{0})\right], \\
\boldsymbol{\phi}^{\mathrm{t}}= & {\left[\phi_{+-}, \phi_{-+}, \phi_{+-}, \phi_{+-}, \phi_{-+}, \phi_{-+},\right.} \\
& \left.\phi_{++}, \phi_{++}, \phi_{--}, \phi_{--}, \phi_{++}, \phi_{--}\right],
\end{aligned}
$$

where $\mathcal{I}$ and $\mathcal{A}(E)$ are $12 \times 12$ matrices; $\mathcal{I}_{v v^{\prime}}=\delta_{v v^{\prime}}(1-$ $\left.\delta_{v 11}-\delta_{v 12}\right)$, and components of $\mathcal{A}(E)$ are integrals over $\mathbf{k}$ space. The integration in Eq. (D22) is performed by applying the Gaussian quadrature rule to discretize $\mathbf{k}$ integrals. It is worth noting that $u_{\mathbf{0 0}}^{ \pm \pm} \psi_{ \pm \pm}(\mathbf{0})$ is finite for the self-consistent solution, while $\psi_{ \pm \pm}(\mathbf{0})=\mathbf{0}$ because of $u_{\mathbf{0 0}}^{ \pm \pm}=\infty$.

The substitution of the obtained $\psi$ to Eq. (D22) provides the wave function for the two-magnon scattering. The value of $a$ is extracted from the wave function by multiplying both sides of Eq. (D22) by $\phi_{m_{1} m_{2}}^{*}$ and taking the sum over $m_{1}$ and $m_{2}$,

$$
\begin{aligned}
\sum_{m_{1}, m_{2}} & \phi_{m_{1} m_{2}}^{*} \psi_{m_{1} m_{2}}(\mathbf{r}) \\
= & 1+\int_{\mathbf{k}} \sum_{m_{1}, m_{2}, m_{1}^{\prime}, m_{2}^{\prime}} \sum_{\mathbf{e}, \mathbf{e}^{\prime}} \phi_{m_{1} m_{2}}^{*}\left[e^{i \mathbf{k} \cdot \mathbf{r}}[G(\mathbf{k})]_{m_{1} m_{2} ; m_{1}^{\prime} m_{2}^{\prime}}\right. \\
& \left.+e^{-i \mathbf{k} \cdot \mathbf{r}}[G(\mathbf{k})]_{m_{2} m_{1} ; m_{1}^{\prime} m_{2}^{\prime}}\right] e^{-i \mathbf{k} \cdot \mathbf{e}} u_{\mathbf{e e}^{\prime}}^{m_{1}^{\prime} m_{2}^{\prime}} \psi_{m_{1}^{\prime} m_{2}^{\prime}}\left(\mathbf{e}^{\prime}\right), \\
& \stackrel{\bar{r} \rightarrow \infty}{\longrightarrow} 1-\frac{a}{\bar{r}},
\end{aligned}
$$

where $\bar{r}=|\overline{\mathbf{r}}|$ and $\overline{\mathbf{r}}=\left(\sqrt{\frac{m_{x}}{m_{z}}} r_{x}, \sqrt{\frac{m_{x}}{m_{z}}} r_{y}, \frac{r_{z}}{2}\right)$ with $\mathbf{r}=r_{x} \mathbf{e}_{x}+$ $r_{y} \mathbf{e}_{y}+r_{z} \mathbf{e}_{z}$. The divergent behavior of the Green's function in the infrared limit,

$$
\sum_{m_{1}, m_{2}} \phi_{m_{1} m_{2}}^{*}[G(\mathbf{k})]_{m_{1} m_{2} ; m_{1}^{\prime} m_{2}^{\prime}} \stackrel{\overline{\mathbf{k}} \rightarrow 0}{\longrightarrow}-\frac{m_{z}}{\overline{\mathbf{k}}^{2}} \delta_{m_{1} m_{1}^{\prime}} \delta_{m_{2} m_{2}^{\prime}},
$$

determines the asymptotic behavior of the integral in Eq. (D24). The definition of $\overline{\mathbf{k}}$ is given in Eq. (4) of the main text,

$$
\begin{aligned}
\overline{\mathbf{k}} & =\left(\bar{k}_{x}, \bar{k}_{y}, \bar{k}_{z}\right) \\
& =\left[\sqrt{\frac{m_{z}}{m_{x}}}\left(k_{1}-\frac{k_{3}}{2}\right), \quad \sqrt{\frac{m_{z}}{m_{x}}}\left(k_{2}-\frac{k_{3}}{2}\right), k_{3}\right] .
\end{aligned}
$$

After changing the variables and extending the integration interval to $[-\infty, \infty]$, we obtain the following asymptotic behavior for the integral in Eq. (D24),

$$
\begin{aligned}
\int_{\mathbf{k}} \frac{e^{i \mathbf{k} \cdot \mathbf{r}}}{\overline{\mathbf{k}}^{2}} & =\frac{1}{2 \pi} \frac{m_{x}}{m_{z}} \int_{-\sqrt{\frac{m_{z}}{m_{x}}} \pi}^{\sqrt{\frac{m_{z}}{m_{x}}} \pi} d \bar{k}_{x} \int_{-\sqrt{\frac{m_{z}}{m_{x}}} \pi}^{\sqrt{\frac{m_{z}}{m_{x}}} \pi} d \bar{k}_{y} \int_{-\pi}^{\pi} d \bar{k}_{z} \frac{e^{i \overline{\mathbf{k}} \cdot \overline{\mathbf{r}}}}{\overline{\mathbf{k}}^{2}} \\
& \simeq \frac{m_{x}}{m_{z}} \frac{1}{4 \pi \bar{r}}
\end{aligned}
$$

which leads to a simple expression for the $s$-wave scattering length,

$$
\begin{aligned}
a & =\frac{m_{x}}{4 \pi} \sum_{m_{1}, m_{2}} \sum_{\mathbf{e}, \mathbf{e}^{\prime}} \phi_{m_{1} m_{2}}^{*}\left\{\psi_{m_{1} m_{2}}\left(\mathbf{e}^{\prime}\right)+\psi_{m_{2} m_{1}}\left(\mathbf{e}^{\prime}\right)\right\} u_{\mathbf{e}^{\prime}}^{m_{1} m_{2}} \\
& =\frac{m_{x}}{4 \pi} \sum_{m_{1}, m_{2}} \sum_{\mathbf{e}, \mathbf{e}^{\prime}} \psi_{m_{1} m_{2}}\left(\mathbf{e}^{\prime}\right) u_{\mathbf{e e}^{\prime}}^{m_{1} m_{2}}
\end{aligned}
$$

The second line is obtained from the first one by using $\phi_{m_{1} m_{2}}=1 / 2$.

Finally, for the two-magnon bound states, we set $E<$ $2 \mathcal{E}_{0}$ and $\phi_{m_{1} m_{2}}=0$ in Eq. (D22). Then, we can obtain the two-magnon bound-state energy $E$ by numerically solving $\operatorname{det}[\mathcal{I}-\mathcal{A}(E)]=0$ instead of Eq. (D23). Its binding energy is provided by $\Delta=E-2 \mathcal{E}_{0}$. 


\section{Three-magnon problem}

The Lippmann-Schwinger equation for the three-magnon problem is derived in the same way as in the previous cases. We introduce the real-space representation of the threemagnon wave function and its Fourier transform,

$$
\begin{aligned}
\Psi\left(\mathbf{r}_{1}, \mathbf{r}_{2}, \mathbf{r}_{3}\right) & =\left\langle\emptyset\left|a_{\mathbf{r}_{3}} a_{\mathbf{r}_{2}} a_{\mathbf{r}_{1}}\right| \Psi\right\rangle, \\
\tilde{\Psi}_{\mathbf{m}}\left(\mathbf{k}_{1}, \mathbf{k}_{2}, \mathbf{k}_{3}\right) & =\left[\prod_{\nu=1}^{3} \sum_{\mathbf{r}_{v} \in m_{v}}\right] e^{-i \sum_{v^{\prime}=1}^{3} \mathbf{k}_{v^{\prime}} \cdot \mathbf{r}_{v^{\prime}}} \Psi\left(\mathbf{r}_{1}, \mathbf{r}_{2}, \mathbf{r}_{3}\right),
\end{aligned}
$$

$$
\begin{aligned}
\psi_{\mathbf{m}}(\mathbf{r} ; \mathbf{k}) & =\frac{1}{L^{3}} \sum_{\mathbf{k}^{\prime}} e^{i \mathbf{k}^{\prime} \cdot \mathbf{r}} \tilde{\Psi}_{\mathbf{m}}\left(-\mathbf{k}-\mathbf{k}^{\prime}, \mathbf{k}^{\prime}, \mathbf{k}\right) \\
& =e^{-i \mathbf{k} \cdot \mathbf{r}} \psi_{\left(m_{2}, m_{1}, m_{3}\right)}(-\mathbf{r} ; \mathbf{k})
\end{aligned}
$$

with $\mathbf{m}=\left(m_{1}, m_{2}, m_{3}\right)$. Note that the zero center-of-mass momentum condition, $\mathbf{k}_{1}+\mathbf{k}_{2}+\mathbf{k}_{3}=\mathbf{0}$, and the exchange symmetry of bosons are imposed in the above transformation. In this way, we obtain a linear set of equations for the threemagnon problem,

$$
\begin{aligned}
\psi_{\mathbf{m}}(\mathbf{r} ; \mathbf{k})= & \int_{\mathbf{k}^{\prime}} \sum_{m_{1}^{\prime}, m_{2}^{\prime}, m_{3}^{\prime}} \sum_{\mathbf{e}, \mathbf{e}^{\prime}}\left[\left\{e^{i \mathbf{k}^{\prime} \cdot \mathbf{r}}\left[G\left(\mathbf{k}^{\prime}, \mathbf{k}\right)\right]_{m_{1} m_{2} m_{3} ; m_{1}^{\prime} m_{2}^{\prime} m_{3}^{\prime}}+e^{-i\left(\mathbf{k}^{\prime}+\mathbf{k}\right) \cdot \mathbf{r}}\left[G\left(\mathbf{k}^{\prime}, \mathbf{k}\right)\right]_{m_{2} m_{1} m_{3} ; m_{1}^{\prime} m_{2}^{\prime} m_{3}^{\prime}}\right\} e^{-i \mathbf{k}^{\prime} \cdot \mathbf{e}} u_{\mathbf{e e}^{\prime}}^{m_{1}^{\prime} m_{2}^{\prime}} \psi_{\left(m_{1}^{\prime}, m_{2}^{\prime}, m_{3}^{\prime}\right)}\left(\mathbf{e}^{\prime} ; \mathbf{k}\right)\right. \\
& +\left[\left\{e^{i \mathbf{k}^{\prime} \cdot \mathbf{r}}\left[G\left(\mathbf{k}^{\prime}, \mathbf{k}\right)\right]_{m_{1} m_{2} m_{3} ; m_{1}^{\prime} m_{2}^{\prime} m_{3}^{\prime}}+e^{-i\left(\mathbf{k}^{\prime}+\mathbf{k}\right) \cdot \mathbf{r}}\left[G\left(\mathbf{k}^{\prime}, \mathbf{k}\right)\right]_{m_{2} m_{1} m_{3} ; m_{1}^{\prime} m_{2}^{\prime} m_{3}^{\prime}}\right\} e^{-i \mathbf{k} \cdot \mathbf{e}}\right. \\
& \left.\left.+\left\{e^{i \mathbf{k}^{\prime} \cdot \mathbf{r}}\left[G\left(\mathbf{k}^{\prime}, \mathbf{k}\right)\right]_{m_{1} m_{2} m_{3} ; m_{3}^{\prime} m_{2}^{\prime} m_{1}^{\prime}}+e^{-i\left(\mathbf{k}^{\prime}+\mathbf{k}\right) \cdot \mathbf{r}}\left[G\left(\mathbf{k}^{\prime}, \mathbf{k}\right)\right]_{m_{2} m_{1} m_{3} ; m_{3}^{\prime} m_{2}^{\prime} m_{1}^{\prime}}\right\} e^{i\left(\mathbf{k}^{\prime}+\mathbf{k}\right) \cdot \mathbf{e}}\right] u_{\mathbf{e e}^{\prime}}^{m_{1}^{\prime} m_{3}^{\prime}} \psi_{\left(m_{1}^{\prime}, m_{3}^{\prime}, m_{2}^{\prime}\right)}\left(\mathbf{e}^{\prime} ; \mathbf{k}^{\prime}\right)\right],
\end{aligned}
$$

where the propagator matrix $G\left(\mathbf{k}^{\prime}, \mathbf{k}\right)$ is defined as

$$
\begin{aligned}
& {\left[G^{-1}\left(\mathbf{k}^{\prime}, \mathbf{k}\right)\right]_{m_{1} m_{2} m_{3} ; m_{1}^{\prime} m_{2}^{\prime} m_{3}^{\prime}}} \\
& \quad \equiv E \delta_{m_{1} m_{1}^{\prime}} \delta_{m_{2} m_{2}^{\prime}} \delta_{m_{3} m_{3}^{\prime}}-\varepsilon_{-\left(\mathbf{k}^{\prime}+\mathbf{k}\right) m_{1} m_{1}^{\prime}} \delta_{m_{2} m_{2}^{\prime}} \delta_{m_{3} m_{3}^{\prime}} \\
& \quad-\varepsilon_{\mathbf{k}^{\prime} m_{2} m_{2}^{\prime}} \delta_{m_{3} m_{3}^{\prime}} \delta_{m_{1} m_{1}^{\prime}}-\varepsilon_{\mathbf{k} m_{3} m_{3}^{\prime}} \delta_{m_{1} m_{1}^{\prime}} \delta_{m_{2} m_{2}^{\prime}} .
\end{aligned}
$$

The 24 unknown functions and the interaction matrix elements acting on them are summarized as

$$
\begin{aligned}
\psi_{(+,-, m)}\left(\mathbf{e}_{z} ; \mathbf{k}\right) & \Rightarrow u_{\mathbf{e}_{z} \mathbf{e}_{z}}^{+-}=u_{\|}, \quad u_{\left(-\mathbf{e}_{z}\right)\left(+\mathbf{e}_{z}\right)}^{+-}=v_{1}, \\
\psi_{(-,+, m)}\left(\mathbf{e}_{z} ; \mathbf{k}\right) & \Rightarrow u_{\mathbf{e}_{z} \mathbf{e}_{z}}^{-+}=u_{\|}, \quad u_{\left(-\mathbf{e}_{z}\right)\left(+\mathbf{e}_{z}\right)}^{-+}=v_{1}, \\
\psi_{(+,-, m)}\left(\mathbf{e}_{x, y} ; \mathbf{k}\right) & \Rightarrow u_{\mathbf{e}_{x, y}^{+-} \mathbf{e}_{x, y}}=u_{\perp}, \\
\psi_{(-,+, m)}\left(\mathbf{e}_{x, y} ; \mathbf{k}\right) & \Rightarrow u_{\mathbf{e}_{x, y}}^{-+} \mathbf{e}_{x, y}=u_{\perp}, \\
\psi_{(+,+, m)}\left(\mathbf{e}_{x, y}+\mathbf{e}_{z} ; \mathbf{k}\right) & \Rightarrow u_{\left(\mathbf{e}_{x, y}+\mathbf{e}_{z}\right)\left(\mathbf{e}_{x, y}+\mathbf{e}_{z}\right)}^{++}=-u_{\perp}, \\
\psi_{(-,-, m)}\left(\mathbf{e}_{x, y}-\mathbf{e}_{z} ; \mathbf{k}\right) & \Rightarrow u_{\left(\mathbf{e}_{x, y}-\mathbf{e}_{z}\right)\left(\mathbf{e}_{x, y}-\mathbf{e}_{z}\right)}^{-}=-u_{\perp}, \\
\psi_{(+,+, m)}(\mathbf{0} ; \mathbf{k}) & \Rightarrow u_{\mathbf{0 0}}^{++}=\mathrm{U}=\infty, \\
\psi_{(-,-, m)}(\mathbf{0} ; \mathbf{k}) & \Rightarrow u_{\mathbf{0 0}}^{--}=\mathrm{U}=\infty,
\end{aligned}
$$

where $m= \pm$. Note that the exchange symmetry of bosons implies $\psi_{\left(m_{1}, m_{2}, m_{3}\right)}(\mathbf{r} ; \mathbf{k})=e^{-i \mathbf{k} \cdot \mathbf{r}} \psi_{\left(m_{2}, m_{1}, m_{3}\right)}(-\mathbf{r} ; \mathbf{k})$ and thus $\psi_{(+,-, m)}\left(-\mathbf{e}_{z} ; \mathbf{k}\right)=e^{i \mathbf{k} \cdot \mathbf{e}_{z}} \psi_{(-,+, m)}\left(\mathbf{e}_{z} ; \mathbf{k}\right)$, and $\psi_{(-,+, m)}\left(-\mathbf{e}_{z} ; \mathbf{k}\right)=e^{i \mathbf{k} \cdot \mathbf{e}_{z}} \psi_{(+,-, m)}\left(\mathbf{e}_{z} ; \mathbf{k}\right)$. Similar to the two-magnon problem, $u_{\mathbf{0 0}}^{ \pm \pm} \psi_{( \pm, \pm, m)}(\mathbf{0} ; \mathbf{k})$ is assumed to be finite, while $\psi_{( \pm, \pm, m)}(\mathbf{0} ; \mathbf{k})=\mathbf{0}$. The self-consistency of this assumption is confirmed by the numerical solutions of Eq. (D32).

To reduce the computational cost, we exploit the symmetry of the wave function $\psi_{\mathbf{m}}(\mathbf{r} ; \mathbf{k})$. The propagator has the following symmetry properties inherited from the effective boson Hamiltonian $\mathcal{H}_{\text {eff }}$,

$$
\begin{aligned}
& {\left[G\left(\mathbf{k}^{\prime}, \mathbf{k}\right)\right]_{m_{1} m_{2} m_{3} ; m_{1}^{\prime} m_{2}^{\prime} m_{3}^{\prime}}} \\
& \quad=\left.\left[G\left(\mathbf{k}^{\prime}, \mathbf{k}\right)\right]_{m_{1} m_{2} m_{3} ; m_{1}^{\prime} m_{2}^{\prime} m_{3}^{\prime}}\right|_{\tilde{k}_{x} \leftrightarrow \tilde{k}_{y}, \tilde{k}_{x}^{\prime} \leftrightarrow \tilde{k}_{y}^{\prime}}
\end{aligned}
$$

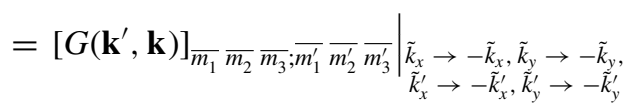

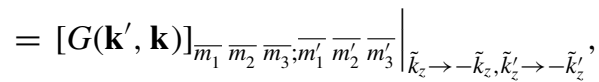

with $\mathbf{k}^{(\prime)}=\frac{1}{2 \pi}\left[\tilde{k}_{x}^{(\prime)} \tilde{\mathbf{G}}_{x}+\tilde{k}_{y}^{(\prime)} \tilde{\mathbf{G}}_{y}+\frac{\tilde{k}_{z}^{(\prime)}}{2} \tilde{\mathbf{G}}_{z}\right]$. By applying these symmetries on the Lippmann-Schwinger equation for the three-magnon problem, we can demonstrate that

$$
\begin{aligned}
& \psi_{\mathbf{m}}(\mathbf{r} ; \mathbf{k}),\left.\quad \psi_{\mathbf{m}}(\mathbf{r} ; \mathbf{k})\right|_{r_{x} \leftrightarrow r_{y}, \tilde{k}_{x} \leftrightarrow \tilde{k}_{y}}, \\
& \left.\psi_{\overline{\mathbf{m}}}(\mathbf{r} ; \mathbf{k})\right|_{r_{x} \rightarrow-r_{x}, r_{y} \rightarrow-r_{y}, \tilde{k}_{x} \rightarrow-\tilde{k}_{x}, \tilde{k}_{y} \rightarrow-\tilde{k}_{y}}, \\
& \left.\psi_{\overline{\mathbf{m}}}(\mathbf{r} ; \mathbf{k})\right|_{r_{z} \rightarrow-r_{z}, \tilde{k}_{z} \rightarrow-\tilde{k}_{z}},
\end{aligned}
$$

with $\overline{\mathbf{m}}=\left(\overline{m_{1}}, \overline{m_{2}}, \overline{m_{3}}\right)$ satisfy the same integral equation. For a nondegenerate eigenstate, the wave function must take the symmetric form,

$$
\begin{aligned}
\psi_{\mathbf{m}}(\mathbf{r} ; \mathbf{k}) & = \pm\left.\psi_{\mathbf{m}}(\mathbf{r} ; \mathbf{k})\right|_{r_{x} \leftrightarrow r_{y}, \tilde{k}_{x} \leftrightarrow \tilde{k}_{y}} \\
& = \pm\left.\psi_{\overline{\mathbf{m}}}(\mathbf{r} ; \mathbf{k})\right|_{r_{x} \rightarrow-r_{x}, r_{y} \rightarrow-r_{y}, \tilde{k}_{x} \rightarrow-\tilde{k}_{x}, \tilde{k}_{y} \rightarrow-\tilde{k}_{y}} \\
& = \pm\left.\psi_{\overline{\mathbf{m}}}(\mathbf{r} ; \mathbf{k})\right|_{r_{z} \rightarrow-r_{z}, \tilde{k}_{z} \rightarrow-\tilde{k}_{z}} .
\end{aligned}
$$

In the calculation, we choose the " + " sign for each symmetry operation because the Efimov states belong to the $s$-wave sector. As in the case of the two-magnon problem, we apply the Gaussian quadrature rule to discretize $\mathbf{k}$ integrals. A bound state with a larger characteristic size (closer to the three-magnon continuum) requires finer momentum space discretization. For the finest momentum space discretization that can be achieved with current supercomputers, we can obtain the energy eigenvalues and the corresponding wave functions for the two lowest-energy states in the Efimov tower.

\section{APPENDIX E: WAVE FUNCTION OF THE EFIMOV STATE IN THE UNITARY LIMIT}

In this Appendix, we consider the wave function of the Efimov state in continuum space [3]. The three-body bound-state 


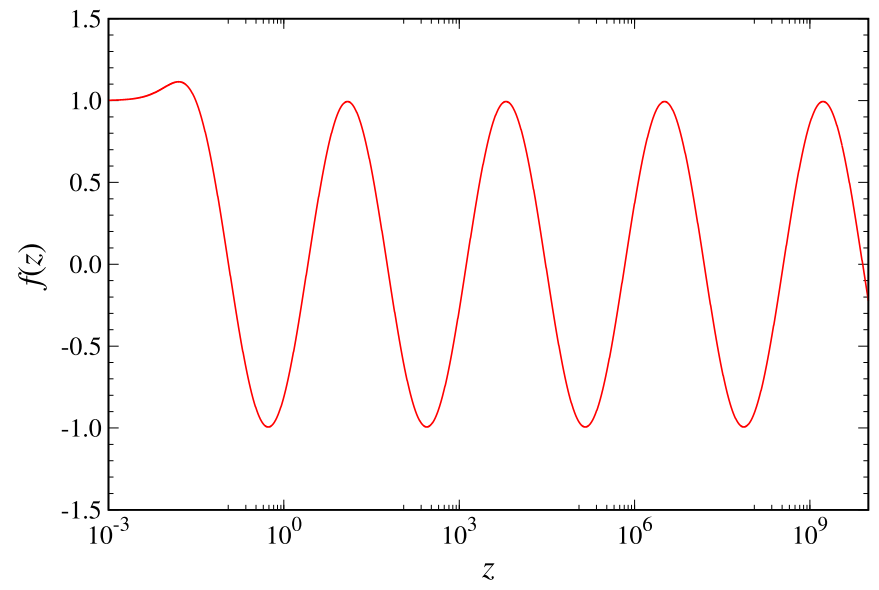

FIG. 9. Universal function $f(z)$ associated with Efimov states. The normalization is chosen to satisfy $f(0)=1$.

problem for bosons with an isotropic mass $m$ can be reduced to a solution of the following Skorniakov-Ter-Martirosian (STM) integral equation,

$$
\begin{aligned}
& {\left[\sqrt{\frac{3 \mathbf{p}^{2}}{4}-m E-i 0^{+}}-\frac{1}{a}\right] \tilde{\phi}(\mathbf{p})} \\
& =\frac{1}{\pi^{2}} \int d \mathbf{q} \frac{1}{\mathbf{p}^{2}+\mathbf{q}^{2}+\mathbf{p} \cdot \mathbf{q}-m E-i 0^{+}} \tilde{\phi}(\mathbf{q}),
\end{aligned}
$$

where $\mathbf{p}$ is the momentum corresponding to $\overline{\mathbf{k}}$ in Eq. (4) and the wave function $\tilde{\phi}(\mathbf{p}) \propto \int d \mathbf{R} \int d \mathbf{r} e^{-i \mathbf{p} \cdot \mathbf{r}} \phi(\mathbf{R}-\mathbf{r} / 2, \mathbf{R}-$ $\mathbf{r} / 2, \mathbf{R}+\mathbf{r} / 2$ ) corresponds to $\psi_{\mathbf{m}}(\mathbf{e} ; \mathbf{k})$ in Eq. (8) of the main text. By setting $E \equiv-\frac{\kappa^{2}}{m}$ and $\tilde{\phi}(\mathbf{p})=\tilde{\phi}(p=|\mathbf{p}|)$, we obtain

$$
\begin{aligned}
& {\left[\sqrt{\frac{3 p^{2}}{4}+\kappa^{2}}-\frac{1}{a}\right] \tilde{\phi}(p)} \\
& =\frac{2}{\pi} \int_{0}^{\infty} d q \frac{q}{p} \ln \left(\frac{p^{2}+q^{2}+p q+\kappa^{2}}{p^{2}+q^{2}-p q+\kappa^{2}}\right) \tilde{\phi}(q) .
\end{aligned}
$$

In the unitary limit $a \rightarrow \infty$, the $n$th bound-state solution is given by

$$
\lim _{n \rightarrow \infty} \kappa_{n}=\frac{\kappa_{*}}{\lambda^{n}} \text { with } \lim _{n \rightarrow \infty} \tilde{\phi}_{n}(p)=\frac{f\left(\sqrt{\frac{3}{4}} \frac{p}{\kappa_{n}}\right)}{\frac{3}{4}\left(\frac{p}{\kappa_{n}}\right)^{2}+1},
$$

where

$$
f(z)=\frac{\sin \left[s_{0} \operatorname{arcsinh}(z)\right]}{s_{0} z} \sqrt{z^{2}+1}
$$

is the universal function plotted in Fig. 9 [35]. The constant $s_{0}=1.00624$ solves

$$
\frac{8}{\sqrt{3} s_{0}} \frac{\sinh \left(\frac{\pi}{6} s_{0}\right)}{\cosh \left(\frac{\pi}{2} s_{0}\right)}=1 .
$$

In Fig. 5 of the main text, we demonstrate that the universal function $f(z)$ well describes the three-magnon wave functions (a)

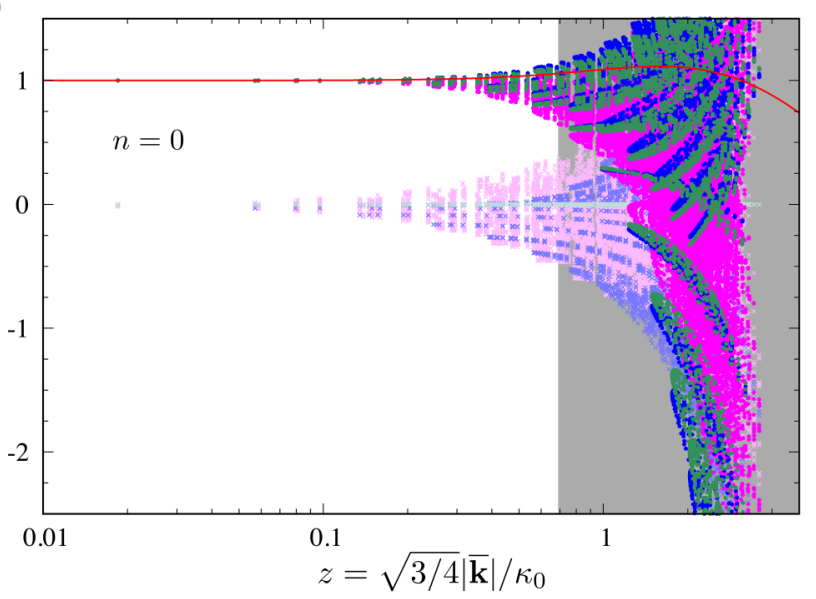

(b)

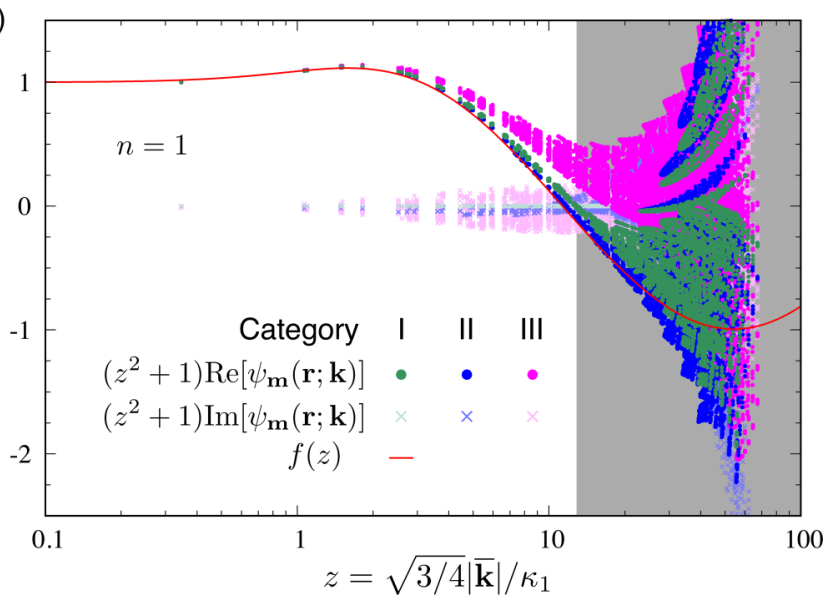

FIG. 10. Wave functions of the two lowest three-magnon bound states at the critical field $\mu_{0} H_{c}=12.91 \mathrm{~T}$. The universal function $f(z)$ and the numerical solutions for (a) the ground state $(n=0)$ and for (b) the first excited state $(n=1)$ are compared as functions of the rescaled wave number $z=\sqrt{3 / 4}|\overline{\mathbf{k}}| / \kappa_{n}$ normalized by $\kappa_{n}$ for each $n$. All the calculated 24 functions are grouped into three categories, I, II, and III, and the common symbols are used for each group as shown in the legend. The gray shaded regions indicate the nonuniversal regime $(|\overline{\mathbf{k}}|>1)$.

especially for the $n=1$ state. Figure 10 now shows the full comparison between the universal function and solutions of the Lippmann-Schwinger equation [Eq. (D32)]. Our numerical calculation provides a set of 24 functions as a solution, which are grouped into three categories as

$$
\begin{aligned}
& \text { I }: \psi_{\left(m, m, m^{\prime}\right)}(\mathbf{0} ; \mathbf{k}), \\
& \text { II }: \psi_{\left(m, \bar{m}, m^{\prime}\right)}\left(\mathbf{e}_{z} ; \mathbf{k}\right), \\
& \text { III }: \psi_{\left(m, \bar{m}, m^{\prime}\right)}\left(\mathbf{e}_{x, y} ; \mathbf{k}\right), \psi_{\left(m, m, m^{\prime}\right)}\left(\mathbf{e}_{x, y}+m \mathbf{e}_{z} ; \mathbf{k}\right) .
\end{aligned}
$$

In addition to category II presented in Fig. 5, the wave functions of categories I and III are also plotted in Fig. 10. All the wave functions show good agreement with the universal function, especially for the $n=1$ state. 
[1] V. Efimov, Energy levels arising from resonant two-body forces in a three-body system, Phys. Lett. B 33, 563 (1970).

[2] E. Nielsen, D. V. Fedorov, A. S. Jensen, and E. Garrido, The three-body problem with short-range interactions, Phys. Rep. 347, 373 (2001).

[3] E. Braaten and H.-W. Hammer, Universality in few-body systems with large scattering length, Phys. Rep. 428, 259 (2006).

[4] F. Ferlaino and R. Grimm, Forty years of Efimov physics: How a bizarre prediction turned into a hot topic, Physics 3, 9 (2010).

[5] H.-W. Hammer and L. Platter, Efimov states in nuclear and particle physics, Annu. Rev. Nucl. Part. Sci. 60, 207 (2010).

[6] P. Naidon and S. Endo, Efimov physics: A review, Rep. Prog. Phys. 80, 056001 (2017).

[7] C. H. Greene, P. Giannakeas, and J. Pérez-Ríos, Universal few-body physics and cluster formation, Rev. Mod. Phys. 89, 035006 (2017).

[8] J. P. D'Incao, Few-body physics in resonantly interacting ultracold quantum gases, J. Phys. B 51, 043001 (2018).

[9] C. Chin, R. Grimm, P. Julienne, and E. Tiesinga, Feshbach resonances in ultracold gases, Rev. Mod. Phys. 82, 1225 (2010).

[10] C. A. Regal, M. Greiner, and D. S. Jin, Observation of Resonance Condensation of Fermionic Atom Pairs, Phys. Rev. Lett. 92, 040403 (2004).

[11] M. W. Zwierlein, C. A. Stan, C. H. Schunck, S. M. F. Raupach, A. J. Kerman, and W. Ketterle, Condensation of Pairs of Fermionic Atoms near a Feshbach Resonance, Phys. Rev. Lett. 92, 120403 (2004).

[12] J. Kinast, S. L. Hemmer, M. E. Gehm, A. Turlapov, and J. E. Thomas, Evidence for Superfluidity in a Resonantly Interacting Fermi Gas, Phys. Rev. Lett. 92, 150402 (2004).

[13] T. Bourdel, L. Khaykovich, J. Cubizolles, J. Zhang, F. Chevy, M. Teichmann, L. Tarruell, S. J. J. M. F. Kokkelmans, and C. Salomon, Experimental Study of the BEC-BCS Crossover Region in Lithium 6, Phys. Rev. Lett. 93, 050401 (2004).

[14] C. Chin, M. Bartenstein, A. Altmeyer, S. Riedl, S. Jochim, J. Hecker Denschlag, and R. Grimm, Observation of the pairing gap in a strongly interacting Fermi gas, Science 305, 1128 (2004).

[15] G. B. Partridge, K. E. Strecker, R. I. Kamar, M. W. Jack, and R. G. Hulet, Molecular Probe of Pairing in the BEC-BCS Crossover, Phys. Rev. Lett. 95, 020404 (2005).

[16] M. W. Zwierlein, J. R. Abo-Shaeer, A. Schirotzek, C. H. Schunck, and W. Ketterle, Vortices and superfluidity in a strongly interacting Fermi gas, Nature (London) 435, 1047 (2005).

[17] N. Navon, S. Piatecki, K. Günter, B. Rem, T. C. Nguyen, F. Chevy, W. Krauth, and C. Salomon, Dynamics and Thermodynamics of the Low-Temperature Strongly Interacting Bose Gas, Phys. Rev. Lett. 107, 135301 (2011).

[18] B. S. Rem, A. T. Grier, I. Ferrier-Barbut, U. Eismann, T. Langen, N. Navon, L. Khaykovich, F. Werner, D. S. Petrov, F. Chevy, and C. Salomon, Lifetime of the Bose Gas with Resonant Interactions, Phys. Rev. Lett. 110, 163202 (2013).

[19] R. J. Fletcher, A. L. Gaunt, N. Navon, R. P. Smith, and Z. Hadzibabic, Stability of a Unitary Bose Gas, Phys. Rev. Lett. 111, 125303 (2013)

[20] P. Makotyn, C. E. Klauss, D. L. Goldberger, E. A. Cornell, and D. S. Jin, Universal dynamics of a degenerate unitary Bose gas, Nat. Phys. 10, 116 (2014).
[21] U. Eismann, L. Khaykovich, S. Laurent, I. Ferrier-Barbut, B. S. Rem, A. T. Grier, M. Delehaye, F. Chevy, C. Salomon, L.-C. $\mathrm{Ha}$, and C. Chin, Universal Loss Dynamics in a Unitary Bose Gas, Phys. Rev. X 6, 021025 (2016).

[22] R. J. Fletcher, R. Lopes, J. Man, N. Navon, R. P. Smith, M. W. Zwierlein, and Z. Hadzibabic, Two- and three-body contacts in the unitary Bose gas, Science 355, 377 (2017).

[23] C. E. Klauss, X. Xie, C. Lopez-Abadia, J. P. D'Incao, Z. Hadzibabic, D. S. Jin, and E. A. Cornell, Observation of Efimov Molecules Created from a Resonantly Interacting Bose Gas, Phys. Rev. Lett. 119, 143401 (2017).

[24] C. Eigen, J. A. P. Glidden, R. Lopes, N. Navon, Z. Hadzibabic, and R. P. Smith, Universal Scaling Laws in the Dynamics of a Homogeneous Unitary Bose Gas, Phys. Rev. Lett. 119, 250404 (2017).

[25] R. J. Fletcher, J. Man, R. Lopes, P. Christodoulou, J. Schmitt, M. Sohmen, N. Navon, R. P. Smith, and Z. Hadzibabic, Elliptic flow in a strongly interacting normal Bose gas, Phys. Rev. A 98 011601(R) (2018).

[26] C. Eigen, J. A. P. Glidden, R. Lopes, E. A. Cornell, R. P. Smith, and Z. Hadzibabic, Universal prethermal dynamics of Bose gases quenched to unitarity, Nature (London) 563, 221 (2018).

[27] Y. Nishida, Y. Kato, and C. Batista, Efimov effect in quantum magnets, Nat. Phys. 9, 93 (2013).

[28] V. Zapf, M. Jaime, and C. D. Batista, Bose-Einstein condensation in quantum magnets, Rev. Mod. Phys. 86, 563 (2014).

[29] S. Onoda and Y. Tanaka, Quantum fluctuations in the effective pseudospin- $\frac{1}{2}$ model for magnetic pyrochlore oxides, Phys. Rev. B 83, 094411 (2011).

[30] S. B. Lee, S. Onoda, and L. Balents, Generic quantum spin ice, Phys. Rev. B 86, 104412 (2012).

[31] J. D. Thompson, P. A. McClarty, D. Prabhakaran, I. Cabrera, T. Guidi, and R. Coldea, Quasiparticle Breakdown and Spin Hamiltonian of the Frustrated Quantum Pyrochlore $\mathrm{Yb}_{2} \mathrm{Ti}_{2} \mathrm{O}_{7}$ in a Magnetic Field, Phys. Rev. Lett. 119, 057203 (2017).

[32] T. Matsubara and H. Matsuda, A lattice model of liquid helium, I, Prog. Theor. Exp. Phys. 16, 569 (1956).

[33] D. Jaksch, C. Bruder, J. I. Cirac, C. W. Gardiner, and P. Zoller, Cold Bosonic Atoms in Optical Lattices, Phys. Rev. Lett. 81, 3108 (1998).

[34] V. Hernandez, J. E. Roman, and V. Vidal, SLEPc: A scalable and flexible toolkit for the solution of eigenvalue problems, ACM Trans. Math. Softw. 31, 351 (2005).

[35] A. O. Gogolin, C. Mora, and R. Egger, Analytical Solution of the Bosonic Three-Body Problem, Phys. Rev. Lett. 100, 140404 (2008).

[36] K. A. Ross, L. Savary, B. D. Gaulin, and L. Balents, Quantum Excitations in Quantum Spin Ice, Phys. Rev. X 1, 021002 (2011).

[37] L. D. C. Jaubert, O. Benton, J. G. Rau, J. Oitmaa, R. R. P. Singh, N. Shannon, and M. J. P. Gingras, Are Multiphase Competition and Order by Disorder the Keys to Understanding $\mathrm{Yb}_{2} \mathrm{Ti}_{2} \mathrm{O}_{7}$ ? Phys. Rev. Lett. 115, 267208 (2015).

[38] D. F. Bowman, E. Cemal, T. Lehner, A. R. Wildes, L. ManginThro, G. J. Nilsen, M. J. Gutmann, D. J. Voneshen, D. Prabhakaran, A. T. Boothroyd, D. G. Porter, C. Castelnovo, K. Refson, and J. P. Goff, Role of defects in determining the magnetic ground state of ytterbium titanate, Nat. Commun. $\mathbf{1 0}$ 637 (2019). 
[39] J. Robert, E. Lhotel, G. Remenyi, S. Sahling, I. Mirebeau, C. Decorse, B. Canals, and S. Petit, Spin dynamics in the presence of competing ferromagnetic and antiferromagnetic correlations in $\mathrm{Yb}_{2} \mathrm{Ti}_{2} \mathrm{O}_{7}$, Phys. Rev. B 92, 064425 (2015).

[40] A. Scheie, J. Kindervater, S. Zhang, H. J. Changlani, G. Sala, G. Ehlers, A. Heinemann, G. S. Tucker, S. M. Koohpayeh, and C. Broholm, Multiphase magnetism in $\mathrm{Yb}_{2} \mathrm{Ti}_{2} \mathrm{O}_{7}$, arXiv:1912.04913.

[41] C. L. Sarkis, J. G. Rau, L. D. Sanjeewa, M. Powell, J. Kolis, J. Marbey, S. Hill, J. A. Rodriguez-Rivera, H. S. Nair, M. J. P. Gingras, and K. A. Ross, Unravelling competing microscopic interactions at a phase boundary: A single crystal study of the metastable antiferromagnetic pyrochlore $\mathrm{Yb}_{2} \mathrm{Ge}_{2} \mathrm{O}_{7}$, arXiv:1912.09448.
[42] M. F. Thorpe, Two-magnon bound state in fcc ferromagnets, Phys. Rev. B 4, 1608 (1971).

[43] B. S. Shastry and B. I. Shraiman, Theory of Raman Scattering in Mott-Hubbard Systems, Phys. Rev. Lett. 65, 1068 (1990).

[44] D. Bertoldo Menezes, A. Reyer, A. Yúksel, B. Bertoldo Oliveira, and M. Musso, Introduction to Terahertz Raman spectroscopy, Spectrosc. Lett. 51, 438 (2018).

[45] A. W. Garrett, S. E. Nagler, D. A. Tennant, B. C. Sales, and T. Barnes, Magnetic Excitations in the $S=1 / 2$ Alternating Chain Compound (VO) ${ }_{2} \mathrm{P}_{2} \mathrm{O}_{7}$, Phys. Rev. Lett. 79, 745 (1997).

[46] D. A. Tennant, C. Broholm, D. H. Reich, S. E. Nagler, G. E. Granroth, T. Barnes, K. Damle, G. Xu, Y. Chen, and B. C. Sales, Neutron scattering study of two-magnon states in the quantum magnet copper nitrate, Phys. Rev. B 67, 054414 (2003). 